\title{
ARTICLE SARI attenuates colon inflammation by promoting STAT1 degradation in intestinal epithelial cells
}

Lei Dai ${ }^{1}$, Yi Liu ${ }^{1}$, Lin Cheng ${ }^{1}$, Huiling Wang ${ }^{1}$, Yi Lin ${ }^{1}$, Gang Shi ${ }^{1}$, Zhexu Dong ${ }^{1}$, Junshu Li ${ }^{1}$, Ping Fan ${ }^{2}$, Qinnan Wang ${ }^{1}$, Xiaolan Su${ }^{1}$, Shuang Zhang ${ }^{3}$, Yang Yang ${ }^{1}$, Xun $\mathrm{Hu}^{2}$, Wei Huang ${ }^{2}$, Zongguang Zhou ${ }^{4}$, Dechao Yu ${ }^{1}$, Christopher Probert ${ }^{5}$, Yuquan Wei ${ }^{1}$ and Hongxin Deng ${ }^{1}$

SARI functions as a suppressor of colon cancer and predicts survival of colon cancer patients, but its role in regulating colitis has not been characterized. Here we show that $\mathrm{SARI}^{-1-}$ mice were highly susceptible to colitis, which was associated with enhanced macrophage infiltration and inflammatory cytokine production. Bone marrow reconstitution experiments demonstrated that disease susceptibility was not dependent on the deficiency of SARI in the immune compartment but on the protective role of SARI in the intestinal epithelial cells (IECS). Furthermore, SARI deficiency enhanced Chemokine (C-C motif) Ligand 2 (CCL2) production and knockout of CCR2 blocks the promoting role of SARI deficiency on colitis. Mechanistically, SARI directly targets and promotes signal transducer and activator of transcription 1 (STAT1) degradation in IECs, followed by persistent inactivation of the STAT1/CCL2 transcription complex. In summary, SARI attenuated colitis in mice by impairing colitis-dependent STAT1/CCL2 transcriptional activation in IECs and macrophages recruitment in colon tissue.

Mucosal Immunology (2019) 12:1130-1140; https://doi.org/10.1038/s41385-019-0178-9

\section{INTRODUCTION}

Inflammatory bowel diseases (IBD), including ulcerative colitis and Crohn's disease, affect the health of over 1 million residents in the USA and 2.5 million in Europe with increasing incidence worldwide, especially in newly industrialized countries (i.e. Asia, South America and Middle East). ${ }^{1}$ Furthermore, recent studies have confirmed the prominent role of "smoldering inflammation" in cancer development, ${ }^{2}$ especially colon cancer. ${ }^{3,4}$ An early clinical study has reported that there is an 18 -fold increase in the risk of developing colorectal cancer in extensive Crohn's colitis and 19fold increase in risk in extensive ulcerative colitis compared with that in the general population. ${ }^{5}$ Thus, colitis is a potential therapeutic target to prevent colon cancer. ${ }^{6}$

During inflammation, several transcription factors, including signal transduction activity and enhancement of transcription (STAT) 3, STAT1 and nuclear factor-KB (NF-KB), function as important regulators in the production of inflammatory factors and the infiltration of inflammatory cells. ${ }^{2,7-9}$ Among them, STAT1 plays an essential role in Toll-like receptor (TLR)-mediated antibody response of marginal zone B cell differentiation during inflammation and infection. ${ }^{10}$ The genetic decrease of STAT1 expression reduces gastric tumorigenesis in gp130 (Y757F/Y757F) mice and coincided with reduced gastritis. ${ }^{11}$ In ulcerative colitis, the expression and activation of STAT1 are greatly increased. ${ }^{12}$ Activation of epithelial STAT1 is elevated in the mucosa of patients with IBD and controls mucosal healing in mice with colitis. $^{13}$ Thus, targeting STAT1 is a potential therapeutic approach for colitis which might reduce colitis-associated cancer. $^{14,15}$

Suppressor of activator protein 1, regulated by IFN (SARl; additionally known as BATF2) was initially identified as an AP1inhibitor, ${ }^{16}$ similar to BATF1 and BATF3. ${ }^{17}$ In cancers, SARI is downregulated and ectopic expression of SARI significantly inhibits progression in a diverse array of cancers. ${ }^{18-21}$ Furthermore, during Mycobacterium tuberculosis and Trypanosoma cruzi infection, SARI controls immunopathology and production of inflammatory and host-protective proteins. ${ }^{22,23}$ A previous study by our group indicated that SARI inhibits angiogenesis by directly targeting ceruloplasmin and inhibiting the HIF-1a/VEGF axis in colon cancer. ${ }^{20}$ However, the role of SARI in colitis development remains elusive.

In this study, acute and chronic colitis models were established in SARI knockout, immuno-reconstructive, and macrophagedepleted mice and the results demonstrated the antiinflammatory role for SARI in mice by promoting STAT1 degradation and negatively regulating STAT1/CCL2 transcriptional activity and macrophages infiltration. These results may provide novel therapeutic targets for colitis, as well as for other inflammatory diseases.

\section{MATERIALS AND METHODS}

Mice and ethics

The SARI wild-type (SARI ${ }^{\mathrm{WT}}$, catalog no. 002448), SARI-deficient $\left(\mathrm{SARI}^{-1-}\right.$, catalog no. 019085; http://jaxmice.jax.org/strain/019085)

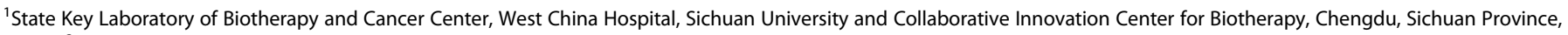

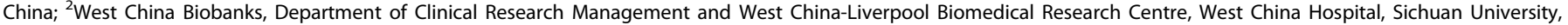

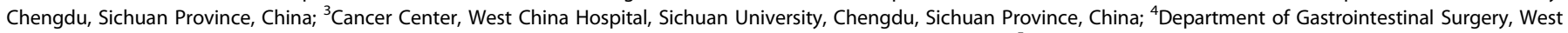

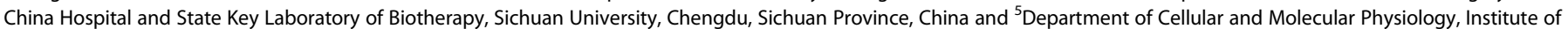
Translational Medicine, University of Liverpool, Liverpool, Merseyside, UK Correspondence: Hongxin Deng (denghongx@scu.edu.cn)
} 
and CCR2-deficient (CCR2 ${ }^{-/-}$, catalog no. 004999; https://www.jax. org/strain/004999) mice were purchased from Jackson Laboratory and used to generate the $\mathrm{SARI}^{-1-} \mathrm{CCR2}^{-/-}$strain. Mouse gene type was determined according to the genotyping protocols supplied by Jackson Laboratory. All mouse care and experiments were carried out in accordance with institutional guidelines concerning animal use and care at Sichuan University.

Induction of colitis

Acute colitis and chronic colitis were induced by dextran sulfate sodium (DSS; molecular mass 36-40 kDa; MP Biologicals, CA, USA) as described by previous studies. ${ }^{24}$ Briefly, acute colitis was induced with $3 \%(\mathrm{w} / \mathrm{v})$ DSS dissolved in sterile, distilled water given to mice ad libitum for the experimental days 1-7, followed by normal drinking water until the end of the experiment. Chronic colitis was induced with $2 \%(\mathrm{w} / \mathrm{v})$ DSS for 7 days, followed by normal drinking water for 14 days. This cycle was repeated thrice and mice were sacrificed after the last DSS cycle.

For macrophages depletion, a single dose of $100 \mu$ l clodronateloaded liposomes (Encapsula Nano Sciences, The Liposomologists, TN, USA) was injected intravenously (i.v.) at 4 days post 3\% DSS treatment. The same volume of phosphate buffered saline was injected as negative control. For CCL2 inhibition, single dose of $100 \mathrm{mg} / \mathrm{kg}$ Bindarit (Selleck, Texas, USA) dissolved in methyl cellulose was intragastrically (i.g.) injected from 2 days before $3 \%$ DSS treatment to 7 days after 3\% DSS treatment. For STAT1 inhibition, single dose of $20 \mathrm{mg} / \mathrm{kg}$ Fludarabine (Selleck) was injected intraperitoneally (i.p.) from 2 days before 3\% DSS treatment to 7 days after 3\% DSS treatment. Further experiments were performed at 14 days post 3\% DSS treatment. The degree of colitis was observed by high-resolution mini-endoscopy (STOKE, Germany)

\section{Clinical scores of colitis}

Scoring for stool consistency and occult blood was done as previously described. ${ }^{24}$ Briefly, stool scores were determined as follows: $0=$ well-formed pellets, $1=$ semi-formed stools that did not adhere to the anus, $2=$ semi-formed stools that adhered to the anus, $3=$ liquid stools that adhered to the anus. Bleeding scores were determined as follows: $0=$ no blood by using Haemoccult (Beckman Coulter), 1 = positive Haemoccult, $2=$ blood traces in stool visible, $3=$ gross rectal bleeding. The highresolution mini-endoscopy (STOKE, Germany) was performed in the $1-4 \mathrm{~cm}$ of mice colon from anus. More than ten images were photographed and scored by two experienced gastro-pathologists in a double-blinded manner.

Histopathological analysis and immunofluorescence staining Paraffin sections were prepared and $4 \mu \mathrm{m}$ slides were used for H\&E staining using a H\&E staining kit (C0105, Beyotime, Beijing, China). Histopathological assessments criteria were: ${ }^{24} 0=$ no evidence of inflammation; $1=$ low level of inflammation with scattered infiltrating mononuclear cells ( $1-2$ foci), $2=$ moderate inflammation with multiple foci, $3=$ high level of inflammation with increased vascular density and marked wall thickening; $4=$ maximal severity of inflammation with transmural leukocyte infiltration and loss of goblet cells. The histopathological score in each mouse was assessed by two experienced gastropathologists based on the H\&E staining of the whole colon.

Paraffin sections were prepared and $4 \mu \mathrm{m}$ slides were used for immunohistochemical (IHC) staining. After dewaxing with xylene and gradient alcohol $(100,95,85$ and $75 \%)$, the slides were performed for antigen retrieval with high temperature and high pressure $(3 \mathrm{~min})$. Then IHC staining kit (SP9001, SP9002, Zsbio, Beijing, China) was used following the instructions. For immunofluorescence staining, after incubating with primary antibodies against F4/80 (1:200; BioLegend, CA, USA), iNOS (1:400, Abcam, Cambridge, UK), CCR2 (1:200, Abcam, Cambridge, UK), EpCAM (1:200, Abcam, Cambridge,
UK), CCL2 (1:200, Abcam, Cambridge, UK) and p-STAT1 (1:200, CST, MA, USA), the sections were then incubated with fluorescein isothiocyanate or tetramethylrhodamine-conjugated secondary antibodies (cat. no. sc-2010, sc-2780, sc-2012, sc-2781; Santa Cruz Biotechnology, Inc., MA, USA). The nucleus was stained with 4',6diamidino-2-phenylindole (DAPI, Thermo Fisher, CA, USA). Images were taken with an Olympus BX51 upright microscope using appropriate fluorescence filters.

Clinical degree of colitis, stool consistency scores, bleeding scores and histopathological scores were calculated by two experienced gastro-pathologists in a double-blinded manner.

Bone marrow chimeras

Recipient 8-week-old SARI ${ }^{\mathrm{WT}}$ and $\mathrm{SARI}^{-/-}$mice were $\gamma$-irradiated with 7.0 Gray (3.5 Gray each time, with a 3-h interval) using the RS200 Biological Irradiator. The irradiated mice were intravenously injected with $2 \times 10^{6} \mathrm{BM}$ cells isolated from SARI ${ }^{\mathrm{WT}}$ and $\mathrm{SARI}^{-1-}$ mice. ${ }^{25}$ Single-cell suspensions were prepared by passing the dissociated cells from the femur and tibia through a $40-\mu \mathrm{m}$ cell strainer and then washing with PBS. Eight weeks post BM injection, blood was collected for gene type determination and the defined mice were used for further experiential study.

\section{Mouse inflammation antibody array}

A mouse inflammation antibody array kit was purchased from RayBiotech (AAM-IFN-1-8, Norcross, GA). Total proteins were collected from SARI ${ }^{\mathrm{WT}}$ and $\mathrm{SARI}^{-1-}$ mice after $3 \%$ DSS treatment for 14 days and used for antibody array analysis. The procedure followed the manufacturer's instructions and ImageJ software was used to quantify the arrays.

\section{Flow cytometry}

For colonic leukocyte isolation, colon samples were sectioned into small pieces and then digested in Digestion Buffer 1 (RPMI 1640 containing $5 \%$ fetal calf serum, $5 \mathrm{mM}$ ethylenediaminetetraacetic acid (EDTA; pH 8.0, Sigma) and $1 \mathrm{mM}$ dithiothreitol (DTT, Sigma)) at $37^{\circ} \mathrm{C}$ for $25 \mathrm{~min}$. After discarding the supernatant, the remaining tissues were digested in Digestion Buffer 2 (RPMI 1640 containing $5 \%$ fetal calf serum, $0.2 \%$ type-IV collagenase (Invitrogen, Carlsbad, CA, USA), and $0.025 \%$ DNase I (Invitrogen)) at $37^{\circ} \mathrm{C}$ for $50 \mathrm{~min}$. The homogenate was then filtered with a 70- $\mu \mathrm{m}$ Nylon cell strainer (BD Biosciences), washed with Washing Buffer 1 (ice-cold PBS (Invitrogen) containing 0.2\% EDTA), and resuspended in Washing Buffer 2 (ice-cold PBS containing 3\% fetal bovine serum (FBS, Gibco) and $0.2 \%$ EDTA). Fluorescentconjugated antibodies used for analyses included CD45 (cat. no. 103116), NK1.1 (cat. no. 108710), CD19 (cat. no. 115507), F4/80 (cat. no. 123108), CD11b (cat. no. 101228), Ly6G (cat. no. 127623), Ly6C (cat. no. 128021) and CD11c (cat. no. 117308) which were obtained from BioLegend and CD4 (cat. no. 553030) and CD8 (cat. no. 100722), which were obtained from BD Bioscience. Labeled cells were analyzed on an NovoCyte flow cytometer (ACEA Biosciences). Data were analyzed with NovoExpress software.

\section{Macrophage depletion}

The SARI ${ }^{-1-}$ mice after 3\% DSS admiration for 4 days were intravenously injected with clodronate-loaded liposomes (Liposoma BV, Amsterdam, Netherlands) in $100 \mu \mathrm{l}$ once. The other $\mathrm{SARI}^{\mathrm{WT}}$ and SARI ${ }^{-1-}$ mice after $3 \%$ DSS admiration for 4 days were intravenously injected with liposomes as a control group. The spleen, lymph node, and colon tissues were collected for further flow cytometry analysis to determine the efficiency of clodronateloaded liposomes on macrophage depletion.

\section{Western blotting}

The entire colon and cultured cells were collected and lysed on ice for $30 \mathrm{~min}$ with the RIPA lysis buffer (Beyotime, Nanjing, China) containing $1 \%$ protease inhibitor cocktail (Merck Millipore). The 
lysate was centrifuged for $15 \mathrm{~min}$ at $12,000 \times g$ at $4{ }^{\circ} \mathrm{C}$ and the supernatants were collected and their concentrations were determined by the Bradford protein assay kit (Thermo Scientific, MA, USA). Equal amounts $(20 \mathrm{mg})$ of protein were loaded into lanes and separated by SDS-PAGE gel electrophoresis, followed by the transfer of proteins onto polyvinylidene difluoride membranes (Merck Millipore). After blocking with TBS/T buffer containing $5 \%$ milk, the membranes were incubated with the primary antibodies against SARI (1:400, Abcam), p-STAT1 (1:1000, CST), STAT1 (1:1200, CST), p-c-Jun (1:1000, CST), p-STAT3 (1:800, CST), STAT3 $(1: 1200$, CST), NF-KB $(1: 1000$, CST), IKK $\beta(1: 1200$, CST), NF-kB p105 (1:1200, CST), p-ІкBa (1:1200, CST), $\beta$-actin (1:50,000, Protein tech), and GAPDH (1:50,000, Protein tech) in 5\% milk TBS/T buffer overnight at $4{ }^{\circ} \mathrm{C}$. Following incubation with horseradish peroxidase-conjugated secondary antibodies (Zsbio, Beijing, China) at room temperature for $1 \mathrm{~h}$, the bands were detected using a chemiluminescent substrate ECL kit (Merck Millipore).

Enzyme-linked immunosorbent assay

The proteins from the entire colon of mice was collected and used to measure the total levels of several cytokines using the mouse CCL2, IL-6, TNF- $\alpha, I L-1 \beta$, and IFN- $\gamma$ ELISA kits (NeoBioscience, Shenzhen, China).

Real-time PCR

Total RNA was extracted from cells using TRIzol reagent (Invitrogen). cDNA was generated from RNA samples $(1 \mu \mathrm{g})$ using the TaKaRa RT reagent kit with the gDNA eraser kit (Takara, Shiga, Japan). For real-time PCR, cDNA was analyzed in triplicate using SYBR Green (Takara). Relative mRNA concentrations were determined by $2^{-\Delta \Delta \mathrm{Ct}}$, where $C_{t}$ is the mean threshold cycle difference after normalization to the levels of U6 mRNA.

\section{Cell culture and treatment}

Human intestinal epithelial cells (HIECs) were purchased from the American Type Culture Collection (ATCC, Manassas, VA, USA) and cultured in Opti-MEM reduced serum medium (Invitrogen) containing $20 \mathrm{mM}$ HEPES (Invitrogen), $10 \mathrm{mM}$ Glutamax (Invitrogen), $10 \mathrm{ng} / \mathrm{ml} \mathrm{EGF} \mathrm{(Peprotech)} \mathrm{and} \mathrm{4 \%} \mathrm{FBS} \mathrm{(Invitrogen).} \mathrm{Cell} \mathrm{line}$ authentication was performed using a short tandem repeat DNA profiling method in our laboratory and the latest verification was done in March 2018. The lentivirus-based short hairpin RNA targeting SARI (lenti-shSARI, 5'-CCCAGGATTTCACAGTCGAA-3') system was used to infect HIECs and the stably infected cells were selected by adding puromycin $(2 \mu \mathrm{g} / \mathrm{ml})$. The selected cells were named as HIECs-shNC and HIECs-shSARI, separately. LPS ( $2 \mu \mathrm{g} / \mathrm{ml}$, Sigma), MG132 (20 $\mu \mathrm{M}$, Selleck) and cycloheximide (CHX, $25 \mu \mathrm{g} / \mathrm{ml}$, Selleck) were added to treat HIECs-shNC and HIECsshSARI separately or jointly.

\section{Coimmunoprecipitation}

For coimmunoprecipitation, the colon tissues of $S A R I^{\mathrm{WT}}$ mice were collected at 14 days post 3\% DSS treatment. The total protein was extracted with RIPA lysis buffer (Beyotime, Nanjing, China) containing $1 \%$ protease inhibitor cocktail (Merck Millipore). The lysate was centrifuged for $15 \mathrm{~min}$ at $12,000 \times \mathrm{g}$ at $4^{\circ} \mathrm{C}$ and the supernatants were collected and their concentrations were determined by the Bradford protein assay kit (Thermo Scientific, MA, USA). Then, $500 \mu \mathrm{g}$ of total protein was added for the direct immunoprecipitation, which was performed according to the instructions of PureProteome ${ }^{\mathrm{TM}}$ Protein A and Protein $\mathrm{G}$ Magnetic Beads (Merck Millipore).

Colonic leukocyte isolation

For mouse colonic leukocyte isolation, the colon samples were sectioned into small pieces and then digested in Digestion Buffer 1 (RPMI 1640 containing 5\% fetal calf serum, 5 mM EDTA (pH 8.0, Sigma) and $1 \mathrm{mM} \mathrm{DTT}$ (Sigma)) at $37^{\circ} \mathrm{C}$ for $25 \mathrm{~min}$. After discarding the supernatant, the remaining tissues were digested in Digestion Buffer 2 (RPMI 1640 containing 5\% fetal calf serum, 0.2\% type-IV collagenase (Invitrogen, Carlsbad, CA, USA), and 0.025\% DNase I (Invitrogen)) at $37^{\circ} \mathrm{C}$ for $50 \mathrm{~min}$. The homogenate was then filtered with a 70- $\mu \mathrm{m}$ Nylon cell strainer (BD Biosciences), washed with ice-cold PBS (Invitrogen) containing 0.2\% EDTA. The final cell pellet was then collected as colonic leukocytes (purity $>90 \%$, shown by flow cytometry detection of CD45 (BioLegend)), which were stored at $-80{ }^{\circ} \mathrm{C}$ for protein extraction.

Intestinal epithelial cells Isolation

For mouse IECs isolation, the colon samples were sectioned into small pieces and washed with PBS containing $100 \mu \mathrm{g} / \mathrm{ml}$ of gentamycin, and immersed in fresh ice-cold PBS containing protease inhibitors and phosphatase inhibitors cocktail (Roche). Colon samples were immersed in $5 \mathrm{ml}$ of ice-cold PBS (Invitrogen) buffer containing $15 \mathrm{mM}$ EDTA and $1 \mathrm{mM}$ dithiothreitol, and the same inhibitors cocktail as above and vortexed at $40 \mathrm{rpm}$ for $50 \mathrm{~min}$ at $4{ }^{\circ} \mathrm{C}$. After standing for $15 \mathrm{~min}$ at $4^{\circ} \mathrm{C}$, the supernatant was collected and passed through a $70 \mu \mathrm{m}$ mesh nylon filter and the filtrate, containing epithelial cells. The cells were washed with ice-cold PBS (Invitrogen) containing 0.2\% EDTA. The final cell pellet was then collected as mouse IECs (purity $>80 \%$, shown by flow cytometry detection of EpCAM (BioLegend)), which were stored at $-80{ }^{\circ} \mathrm{C}$ for protein extraction.

\section{Statistical analysis}

All experiments were repeated 3-5 times and the data are expressed as the mean \pm SD. Statistical analysis was performed by the Student's $t$ tests for comparing two groups and by analysis of variance for multiple group comparisons; $P<0.05$ was considered statistically significant. All calculations were performed using SPSS v17.0 (SPSS Inc., Chicago, IL, USA).

\section{RESULTS}

SARI deficiency promotes colitis in mice

To define the role of SARI in colitis, 3\% DSS-induced acute colonic inflammation model was established and the features (transmural leukocyte infiltration and loss of goblet cells) were consistent with published literature. ${ }^{24}$ Compared with SARI ${ }^{\mathrm{WT}}$ mice, there was significantly more weight loss (Fig. 1a), colon length shortening (Fig. 1b), bleeding (Fig. 1c) and diarrhea (Fig. 1d) in SARI ${ }^{-1-}$ mice in DSS-induced colitis. Representative mini-endoscopic images (Fig. 1e) and histopathological analysis (Fig. 1f) of colon crosssections were taken on days 7, 11, and 14 after induction of acute colitis. SARI ${ }^{-1-}$ mice developed more severe colitis and larger areas of tissue destruction with altered crypt structure accompanied by massive immune cell infiltrates than $S A R I^{\mathrm{WT}}$ mice (Fig. 1e, f). ELISA analysis suggested that deletion of SARI promoted proinflammatory cytokine (IL-6, TNF-a, IL-1 $\beta$, and IFN- $\gamma$; Fig. $1 \mathrm{~g}$ ) expression in acute colitis.

In the $2 \%$ DSS-induced chronic colitis model, more severe colitis was again observed in $S A R I^{-1-}$ mice, as evidenced by shortened colon length (Supplementary Fig. 1a), higher histopathological inflammation score (Supplementary Fig. 1b), and higher proinflammatory cytokine expression (Supplementary Fig. 1c). However, there was no significant difference between SARI ${ }^{W T}$ and $S A R I^{-I-}$ mice in terms of colon length and histopathological analysis after mice recovered from colitis (Supplementary Fig. 1d, e). These results indicate that SARI-deficient mice were hyper-susceptible to colitis-induced inflammation.

SARI deficiency promotes colon inflammation dependent on macrophage recruitment in colon tissue

To evaluate the effect of the inflammatory microenvironment on colitis development, we examined leukocyte infiltration. We found significantly increased macrophage $\left(\mathrm{F} 4 / 80^{+} / \mathrm{CD} 11 \mathrm{~b}^{+}\right)$numbers 

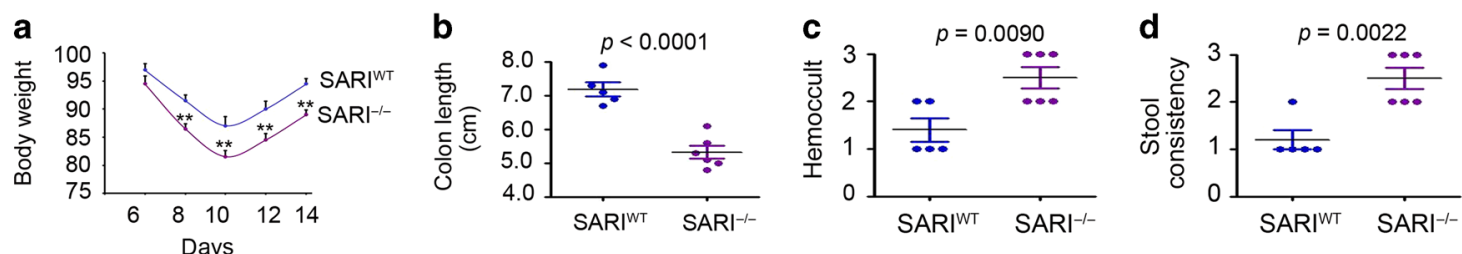

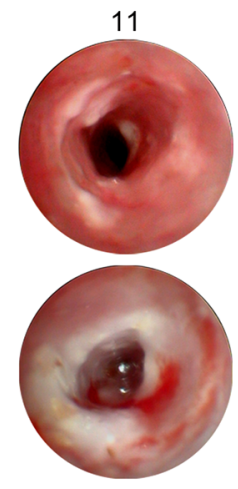

11
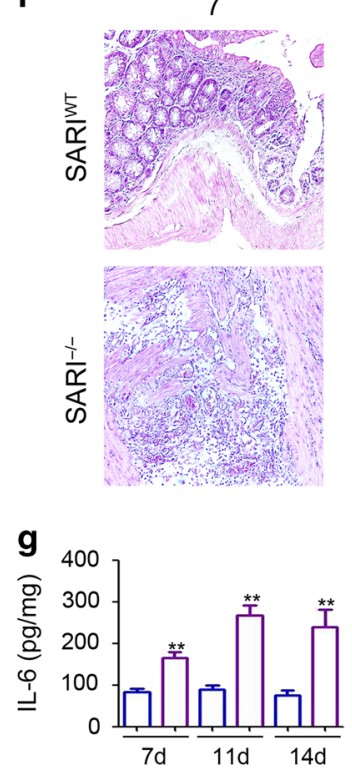
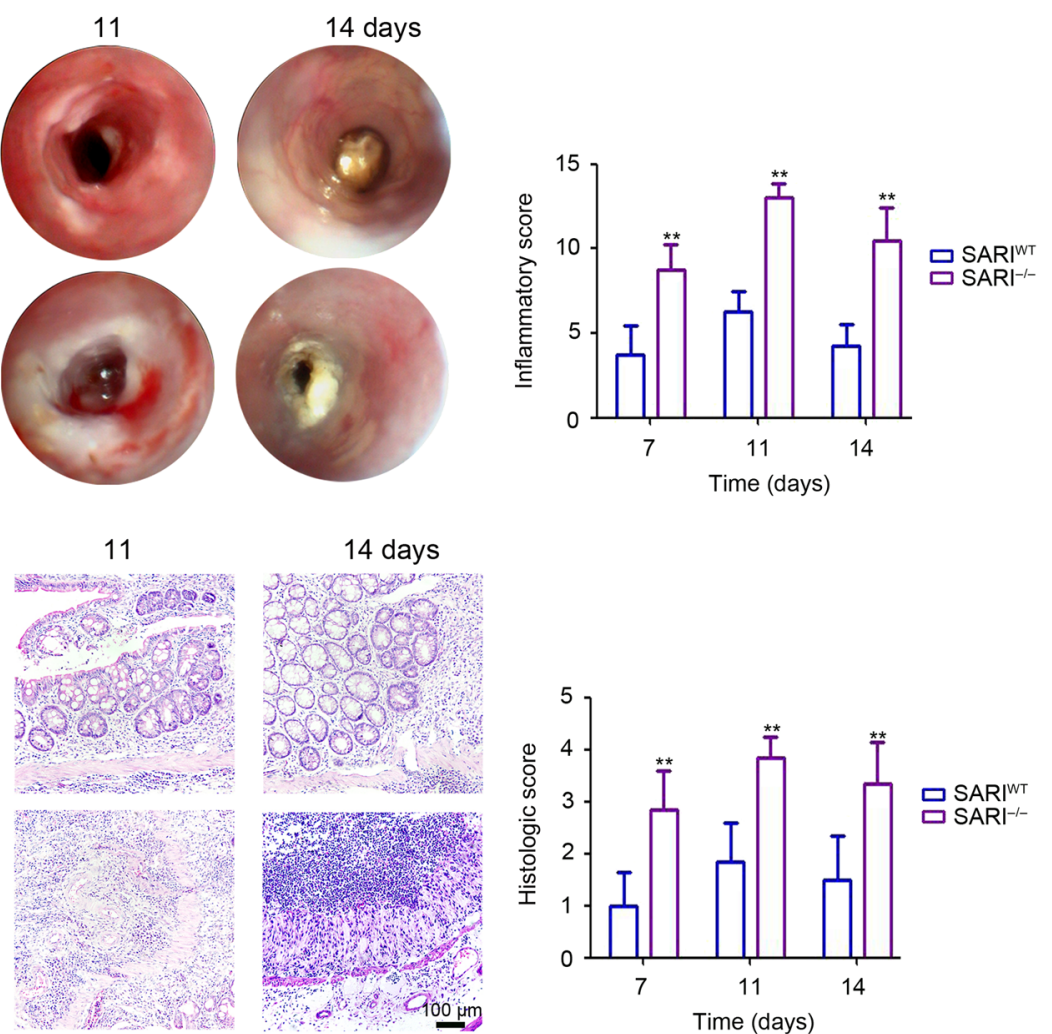

14 days
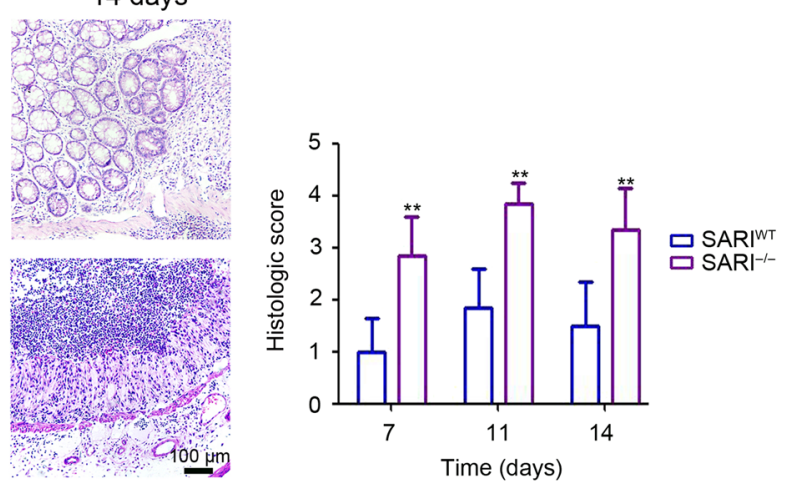

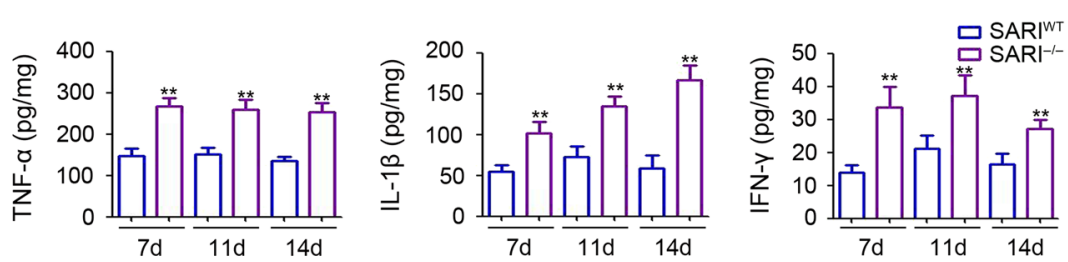

Fig. 1 SARI deficiency promotes acute colitis. a SARI ${ }^{\mathrm{WT}}$ and $S A R I^{-1-}$ mice received $3 \%$ DSS treatment (in drinking water) for $7 \mathrm{~d}$ (acute colitis

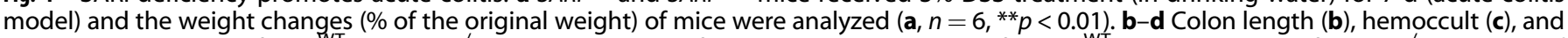

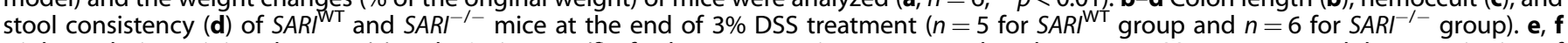

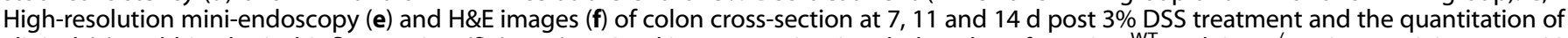

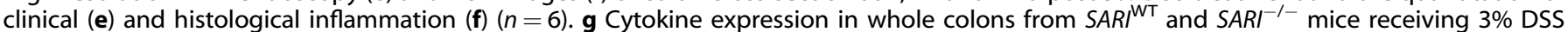
treatment for 7,11 , and $14 \mathrm{~d}$ was determined by ELISA $\left(n=4,{ }^{* *} p<0.01\right)$

and moderately increased neutrophil $\left(\mathrm{Ly}_{6 \mathrm{G}}{ }^{+} / \mathrm{CD} 11 \mathrm{~b}^{+}\right)$and monocytes $\left(\mathrm{Ly}_{6 \mathrm{G}} / \mathrm{G}^{-/ \mathrm{low}} / \mathrm{Ly} 6 \mathrm{C}^{+} / \mathrm{CD} 11 \mathrm{~b}^{+}\right)$counts in the colon sections of $S A R I^{-1-}$ mice compared with those in $S A R I^{\mathrm{WT}}$ mice at $14 \mathrm{~d}$ post $3 \%$ DSS treatment (Fig. 2a). Notably, the number of leukocyte cells did not significantly increase under normal conditions (Fig. 2a). Immunofluorescence staining showed more extensive macrophage infiltration into colon sections and elevated expression of inducible nitric oxide synthase (iNOS) in $S A R I^{-1-}$ mice compared with that in SARI ${ }^{\mathrm{WT}}$ mice in acute colitis (Fig. 2b).

To determine whether macrophages played a crucial role during inflammation regulation by SARI in colitis models, we depleted macrophages in $S A R I^{-1-}$ mice by injection of clodronate-loaded liposomes, which induced macrophage apoptosis (Supplementary Fig. 2a-d). ${ }^{26}$ After liposome treatment, the weight of $S A R I^{-1-}$ mice was restored compared with that in untreated $S A R I^{-1-}$ mice in the acute colitis model (Fig. 2c). The macrophage deletion prevented colon length shortening (Fig. $2 \mathrm{~d}$ ) and improved stool consistency (Fig. 2e) and reduced bleeding (Fig. 2f) in SARI ${ }^{-1-}$ mice with acute colitis. Representative mini-endoscopic (Fig. 2g), histopathological (Fig. 2h) and ELISA (Supplementary Fig. 2e) analyses confirmed the preventative role of macrophage depletion during SARI deficiency-mediated inflammation upregulation. These results demonstrated the crucial role of macrophages during SARIregulated colitis-associated inflammation. 

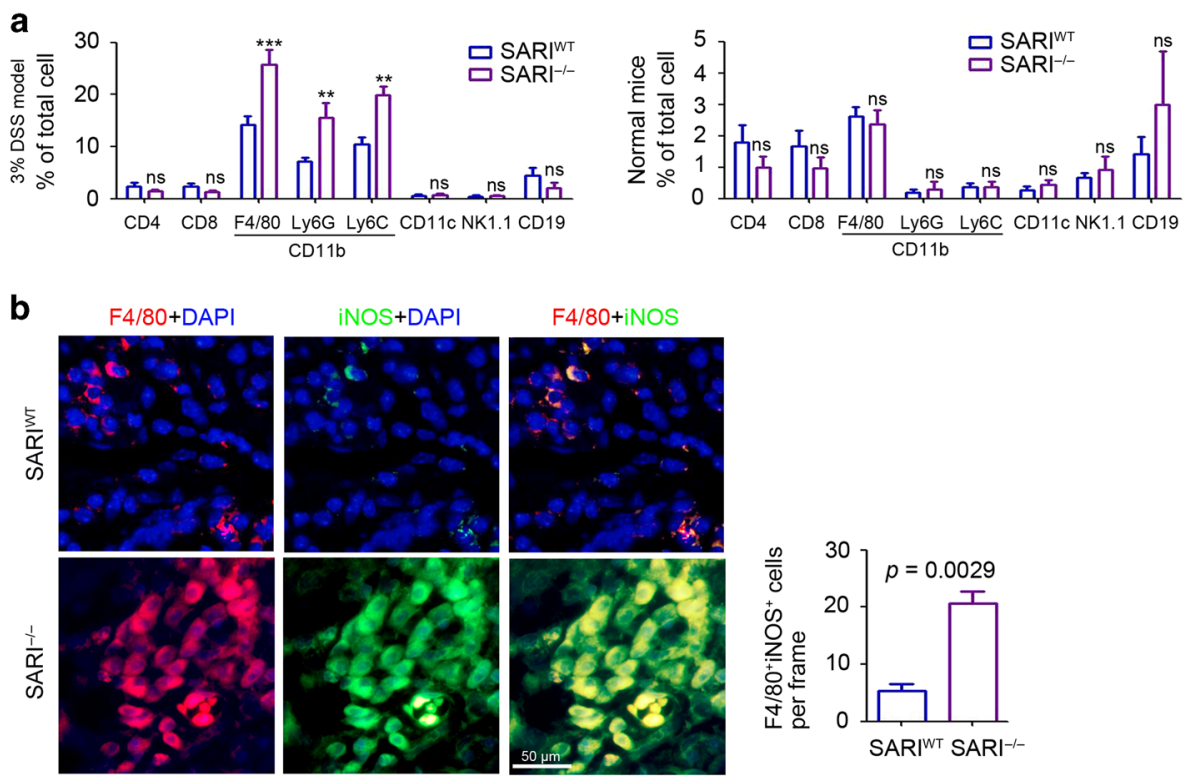

F4/80+iNOS
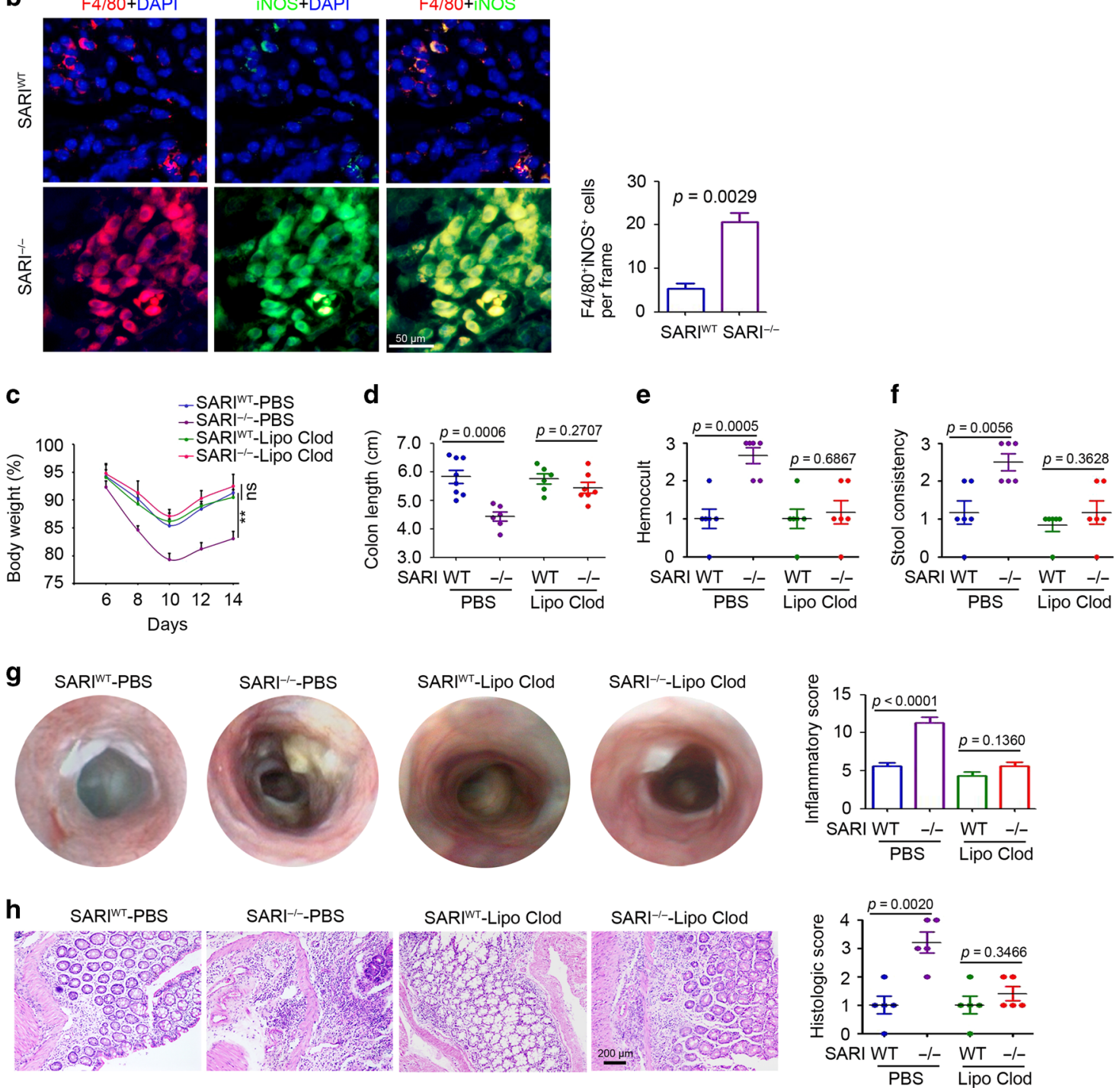

Fig. 2 SARI deficiency promotes colon inflammation dependent on macrophages recruitment. a Flow cytometry analysis of immune cell infiltration in colonic tissues of $S A R I^{\mathrm{WT}}$ and $S A R I^{-1-}$ mice at $14 \mathrm{~d}$ post treatment $3 \%$ DSS treatment in the acute colitis mouse model and normal mice. The percentage of immune cells was analyzed $\left(n=3,{ }^{* *} p<0.01\right.$; ${ }^{* *} p<0.001$; ns, no significant difference; compared with $S A R I^{W T}$ mice). b Representative images of F4/80 (red) and iNOS (green) staining of colon tissues at the end of the 3\% DSS treatment. The cell nuclei were stained with DAPI (blue) $(n=4)$. c Weight change analysis (\% of the original weight) of PBS-injected SARI $I^{\mathrm{WT}}$ and $S A R I^{-/-}$mice and lipo clod-injected SARI $I^{-1-}$ mice (Lipo clod, one treatment) receiving 3\% DSS treatment $\left(n=8\right.$ for SARI ${ }^{\mathrm{WT}}$-PBS; $n=6$ for SARI ${ }^{-1-}$-PBS; $n=6$ for $S A R I^{\mathrm{WT}}$-Lipo clod; $n=7$ for SARI ${ }^{-1-}$-Lipo clod, $\left.{ }^{* *} p<0.01\right)$. d Colon length, hemoccult (e) and stool consistency (f) of mice at the end of $3 \%$ DSS

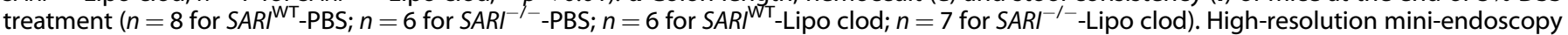
(g) and H\&E images (h) of colon cross-section at 14 days post 3\% DSS treatment and the quantitation of clinical (g) and histological inflammation (h) ( $n=5$ for each group)

SARI deficiency in epithelial cell promotes colon inflammation To investigate the effects of bone marrow (BM)-derived macrophages in $S A R I^{\mathrm{WT}}$ and $S A R I^{-1-}$ mice, chimeric BM mice were established. The irradiated $\mathrm{SARI}^{-1-}$ mice receiving $S A R I^{\mathrm{WT}}$ and $S A R I^{-1-} \mathrm{BM}$ had more severe weight loss, colon length shortening, bleeding and diarrhea compared with that in the irradiated $S A R I^{\mathrm{WT}}$ mice receiving $S A R I^{\mathrm{WT}} \mathrm{BM}$, whereas the irradiated $S A R I^{\mathrm{WT}}$ mice receiving $S A R I^{-1-} \mathrm{BM}$ developed similar colitis, as did the irradiated $S A R I^{\mathrm{WT}}$ mice receiving $S A R I^{\mathrm{WT}}$ BM (Fig. 3a-d). Representative mini-endoscopic (Fig. 3e) and histopathological (Fig. 3f) analyses also confirmed the more severe colitis in the irradiated $S A R I^{-1-}$ mice receiving $S A R I^{\mathrm{WT}}$ and $S A R I^{-1-} \mathrm{BM}$. Further results suggested that colon sections from irradiated $S A R I^{-1-}$ mice receiving $S A R I^{\mathrm{WT}}$ and $S A R I^{-1-} \mathrm{BM}$ were infiltrated with massive 

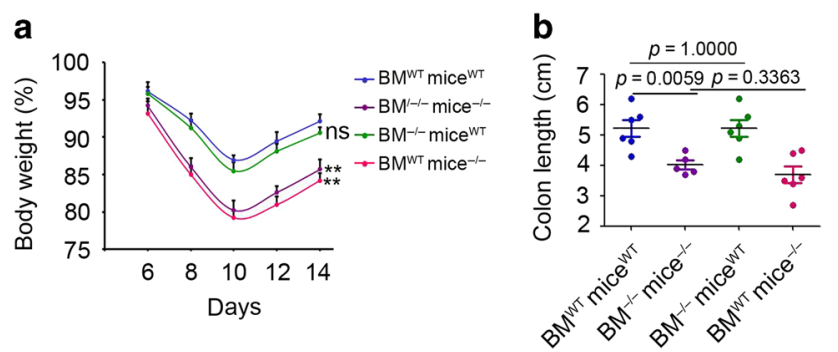

C
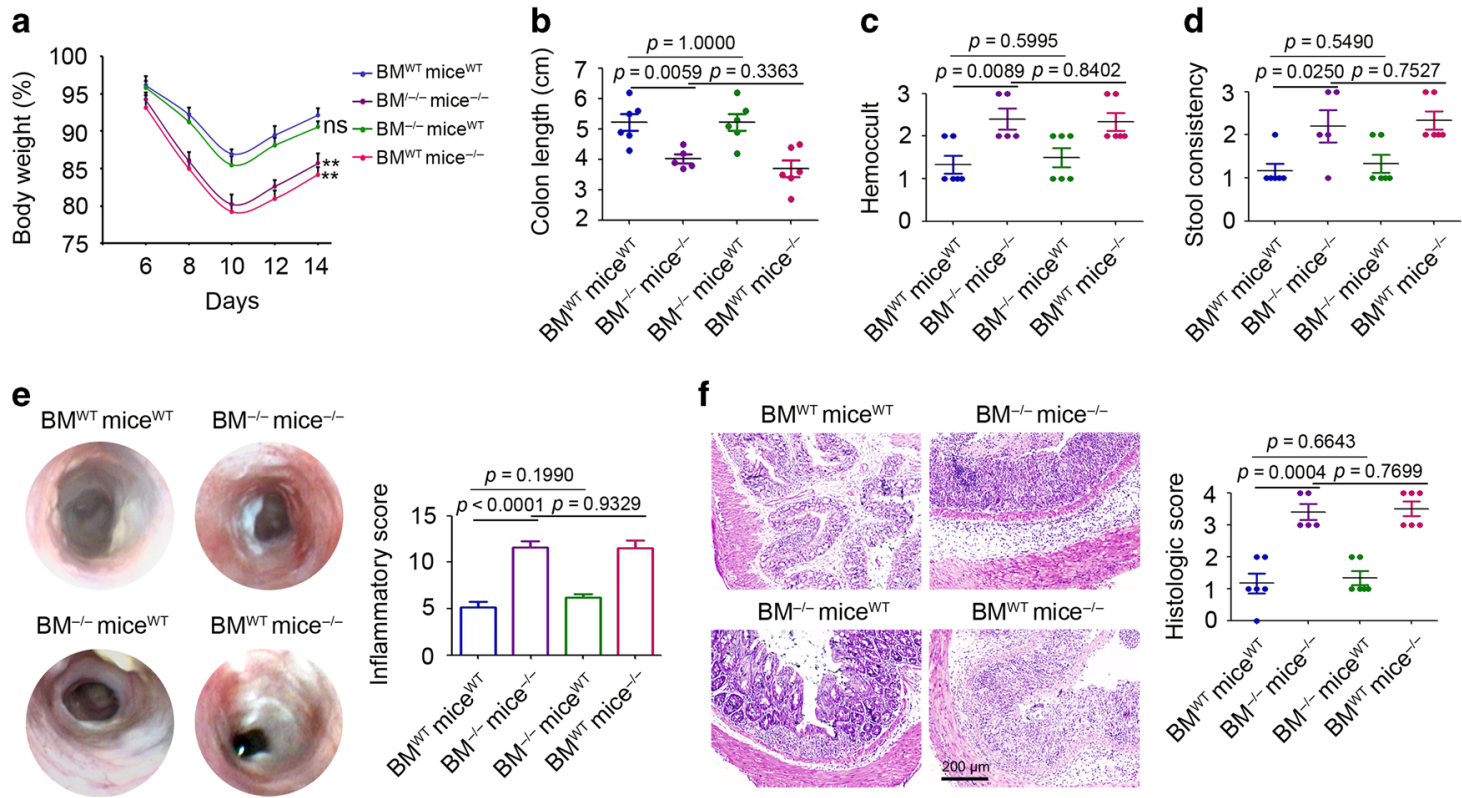

Fig. 3 SARI deficiency in epithelial cell promotes colon inflammation. a Weight change analysis (\% of the original weight), of $S A R I^{W T}$ mice with

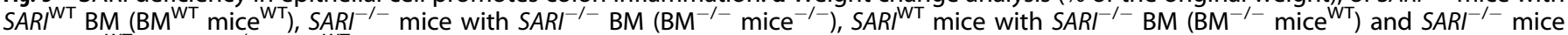
with $S A R I^{\mathrm{WT}} \mathrm{BM}\left(\mathrm{BM}^{-1-}\right.$ mice $\left.{ }^{\mathrm{WT}}\right)$ receiving $3 \%$ DSS in drinking water treatment $\left(n \geq 5,{ }^{* *} p<0.01\right.$; ns, no significant difference, compared with $\mathrm{BM}^{\mathrm{WT}}$ mice ${ }^{\mathrm{WT}}$ group.). Colon length (b), hemoccult (c) and stool consistency (d) of BM chimeras mice receiving $3 \%$ DSS in drinking water ( $n \geq$ 5). High-resolution mini-endoscopy (e) and H\&E images (f) of colon cross-section at the end of treatment and the quantitation of clinical (e) and histological inflammation (f) $(n \geq 5)$

numbers of immune cells and activated macrophages and showed a significant increase in proinflammatory cytokine expression, compared with those in the irradiated $S A R I^{\mathrm{WT}}$ mice receiving $S A R I^{\mathrm{WT}} \mathrm{BM}$, whereas there was no observed difference between the irradiated $S A R I^{\mathrm{WT}}$ mice receiving $S A R I^{\mathrm{WT}}$ and $S A R I^{-1-} \mathrm{BM}$ (Supplementary Fig. 3). These results further demonstrated that SARI deficiency in BM cells has no observed role in the promotion of colitis.

Enhanced CCL2 production in SARI-deficient mice drives acute colitis

Changes in expression of inflammatory cytokines between the colonic tissues of $S A R I^{\mathrm{WT}}$ and $S A R I^{-1-}$ mice with acute colitis were determined. The results showed that the levels of Eotaxin-1, IL-6, IL-12, KC, CCL2, M-CSF, and sTNFRII were increased, whereas that of IL-13 was decreased in $S A R I^{-1-}$ mice with acute colitis relative to the levels found in $S A R I^{\mathrm{WT}}$ mice with acute colitis (Fig. 4a). However, there was no observed difference in inflammatory cytokine expression between the colonic tissues of SARI ${ }^{\mathrm{WT}}$ and $S A R I^{-1-}$ mice without colitis (Supplementary Fig. 4a). ELISA assays further confirmed that CCL2 expression was maintained at a high level and markedly increased in $S A R I^{-1-}$ mice at different time points after 3\% DSS treatment (Fig. 4b). Colonic CCL2 expression in $S A R I^{\mathrm{WT}}$ and $S A R I^{-1-}$ mice without colitis was similar (Supplementary Fig. 4b). The mouse IECs and colonic leukocytes were isolated from colonic tissues of $S A R I^{\mathrm{WT}}$ and $S A R I^{-1-}$ mice receiving $3 \%$ DSS treatment for 14 days. The results indicated that SARI deficiency promotes CCL2 expression in IECs, but not in colonic leukocytes (Fig. 4b). Proinflammatory cytokines expression was upregulated in colonic leukocytes, but not in epithelial cells (Fig. 4c). Notably, depleting macrophages had no significant effect on colonic CCL2 expression (Supplementary Fig. 4c). Moreover, immunofluorescence analysis confirmed that epithelial CCL2 expression was upregulated in $S A R I^{-1-}$ mice (Fig. 4d), and that more CCR2-positive macrophages $\left(\mathrm{F} 4 / 80^{+}\right)$infiltrated into colonic tissue of $S A R I^{-1-}$ mice after inducing colitis (Fig. 4e). To determine whether CCL2 plays a critical role during SARI-mediated colitis regulation, Bindarit, a CCL2-specific inhibitor, ${ }^{27}$ was employed to treat $S A R I^{-1-}$ mice with acute colitis (Supplementary Fig. 4d). ELISA results confirmed the efficient inhibition of colonic CCL2 expression by Bindarit (Supplementary Fig. 4e). Moreover, Bindarit was found to prevent $S A R I^{-1-}$ mice from developing more severe colitis (Supplementary Fig. 4f, g). Representative mini-endoscopic (Fig. 4f) and histopathological staining (Fig. 4g) also confirmed the preventing role of Bindarit in $S A R I^{-1-}$-induced more severe colitis under $3 \%$ DSS treatment. Lower proinflammatory cytokine expression was observed in $S A R I^{-1-}$ mice with Bindarit treatment (Supplementary Fig. 4g). These results demonstrated that CCL2 plays an indispensable role during SARI-regulated colitis.

Knockout of CCR2 blocks the promoting role of SARI deficiency on acute colitis

To further investigate the role of CCL2 during SARI-regulated colitis, $S A R I^{-1-} C C R 2^{-1-}$ and single-gene knockout mice were generated and colitis was induced. SARI deficiency-induced severe weight loss and colon length shortening were additionally attenuated in CCR2 knockout mice (Fig. 5a, b); less severe bleeding and improved stool consistency were detected in $S A R I^{-1-} C C R 2^{-1-}$ and $S A R I^{\mathrm{WT}} C C R 2^{-1-}$ mice relative to $S A R I^{-1-}$ $C C R 2^{\mathrm{WT}}$ mice (Fig. $5 \mathrm{c}$, d). Representative mini-endoscopic images and histopathological analysis of colon cross-sections showed that the knockout of CCR2 attenuated SARI deficiency-mediated colitis (Fig. 5e, f). Furthermore, immunofluorescence staining and ELISA analysis further suggested that fewer activated macrophages infiltrated and lower proinflammatory expression occurred in $S A R I^{-1-} C C R 2^{-1-}$ and $S A R I^{\mathrm{WT}} C C R 2^{-1-}$ mice (Supplementary Fig. 5). Collectively, the above data indicated that knockout of CCR2 blocks the effects of SARI deficiency on colitis.

SARI inhibits CCL2 expression through targeting STAT1 in intestinal epithelial cells

CCL2 mRNA expression was determined by real-time PCR and the results indicated $t$ SARI deficiency promotes the expression of CCL2 at the transcriptional level in colitis mice (Supplementary 
L Dai et al.
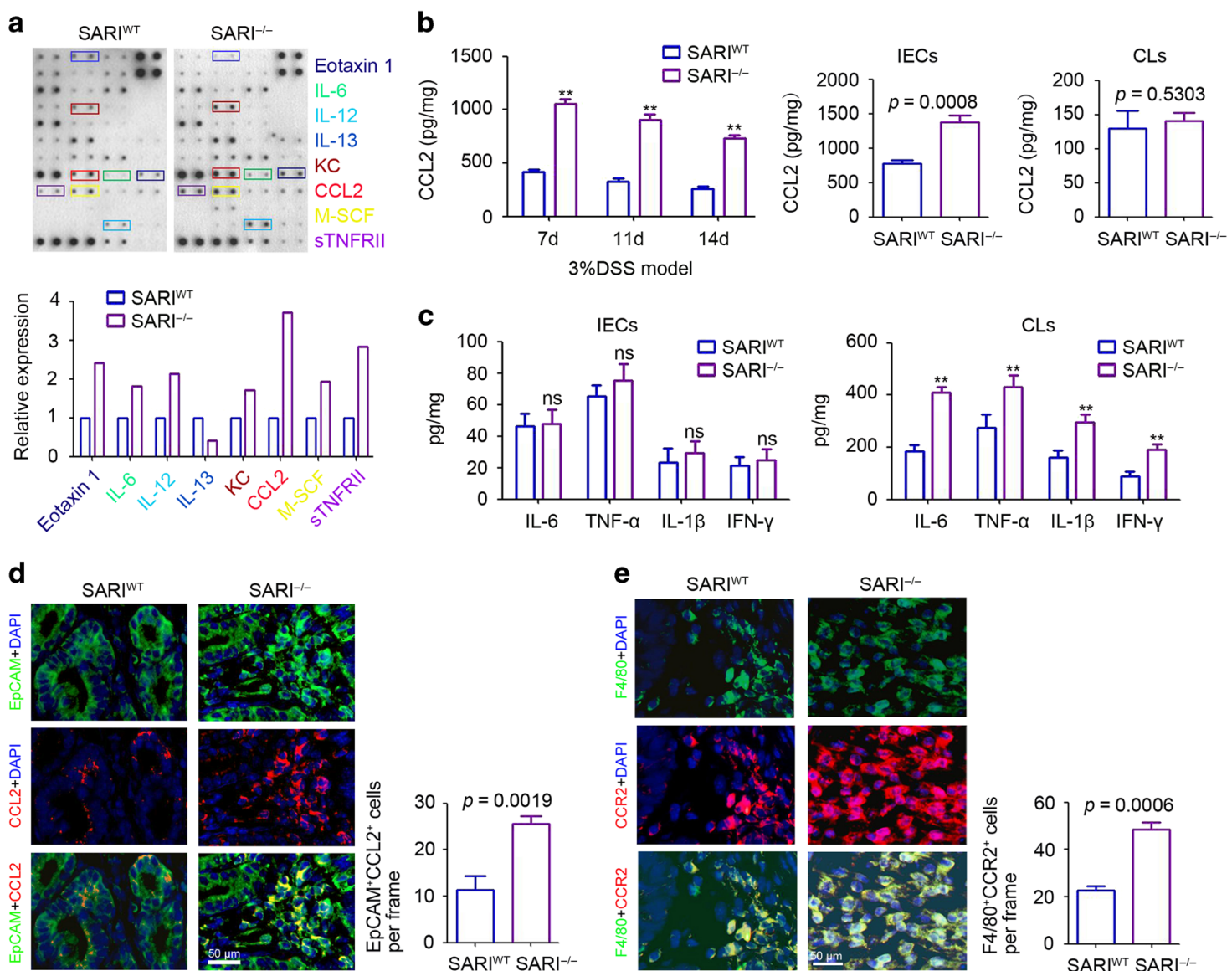

$\mathbf{f}$
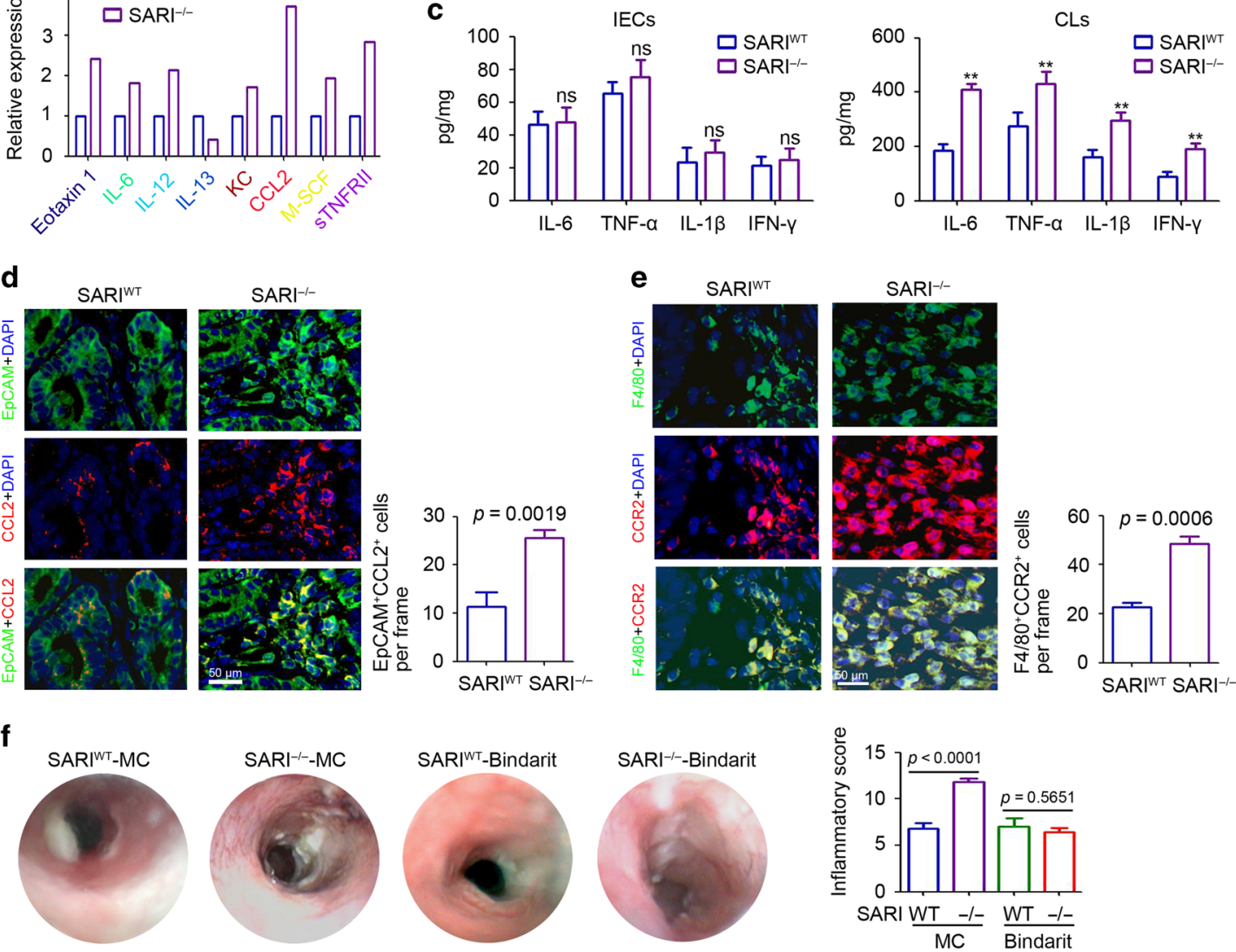

g
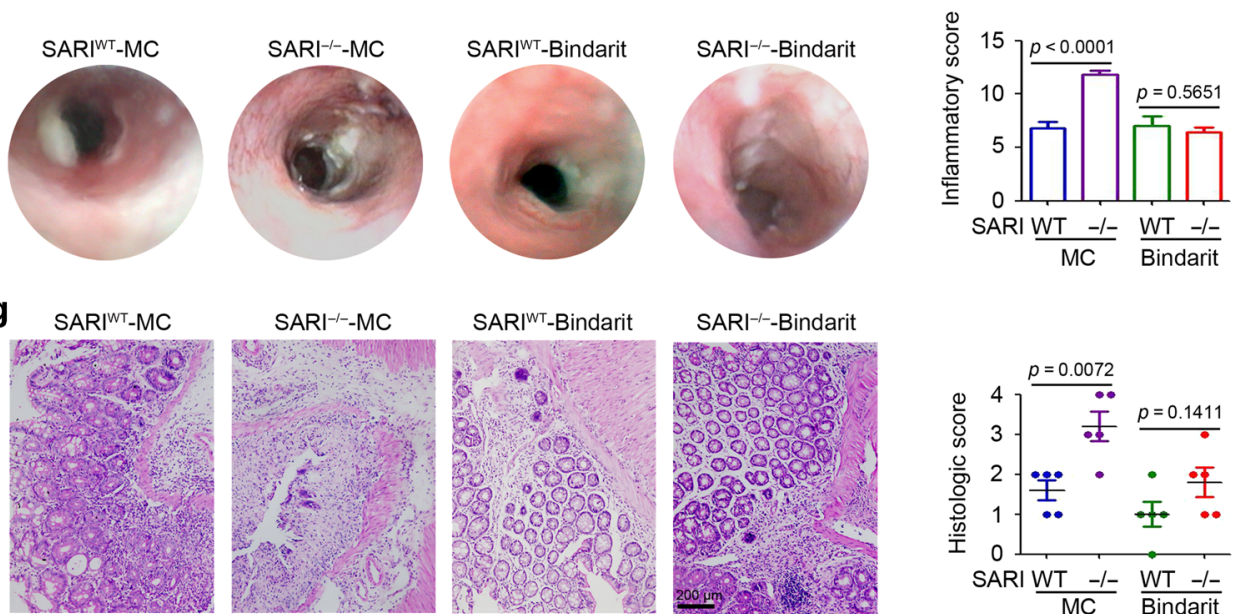

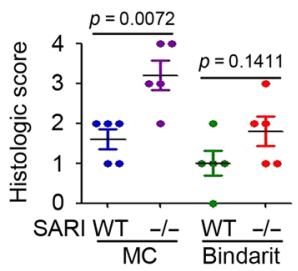

Fig. 4 Enhanced CCL2 production in SARI-deficient mice drives colitis. a Mice cytokine array analysis of the colonic proteins of SARI ${ }^{\mathrm{WT}}$ mice and $S A R I^{-1-}$ mice receiving $3 \%$ DSS treatment for $14 \mathrm{~d}$ and summary of the relative signal intensity of indicated cytokines. $\mathbf{b}$ ELISA analysis of CCL2 expression in colonic tissues at 7, 11 and $14 \mathrm{~d}$ post 3\% DSS treatment and in intestinal epithelial cells (IECs) and colonic leukocytes (CLs) that separated from colonic tissues of SARI ${ }^{\mathrm{WT}}$ and SARI ${ }^{-1-}$ mice at the end of $3 \%$ DSS treatment $\left(n=4,{ }^{* *} p<0.01\right)$. c ELISA analysis of IL-6, TNF$\alpha$, IL-1 $\beta$ and IFN- $\gamma$ expression in intestinal epithelial cells (IECs) and colonic leukocytes (CLs) that separated from colonic tissues of SARI ${ }^{W T}$ and $S A R I^{-1-}$ mice at the end of $3 \%$ DSS treatment $\left(n=4,{ }^{* *} p<0.01 ;\right.$ ns, no significant difference). d Representative images of CCL2 (red) and EpCAM (green) staining of colon tissues. The number of CCL2- and EpCAM-positive cells in per frame were counted and analyzed ( $n=4)$. e Representative images of F4/80 (green) and CCR2 (red) staining of colon tissues. The number of F4/80- and CCR2-positive cells in per frame were counted and analyzed $(n=4)$. High-resolution mini-endoscopy $(\mathbf{f})$ and H\&E images $(\mathbf{g})$ of colon cross-section at the end of treatment and the quantitation of clinical (f) and histological inflammation $(\mathbf{g})(n \geq 4)$

Fig. 6a). To determine the direct target of SARI in the regulation of CCL2 expression, coimmunoprecipitation and mass spectrometry were performed in colonic tissues from colitis SARI ${ }^{\mathrm{WT}}$ mice and indicated that STAT1 is predicted as a direct target of SARI (Fig. 6a). Immunoprecipitation and western blotting showed that
SARI specifically interacts with STAT1, but not STAT3 (Fig. 6b). Next, western blotting was performed to investigate the expression of several inflammation-related transcription factors STAT1, STAT3, and NF-KB ${ }^{12,28,29}$ and higher levels of p-STAT1, p-STAT3, and NF-KB were found in the SARI-deficient mice (Fig. $6 c$; 

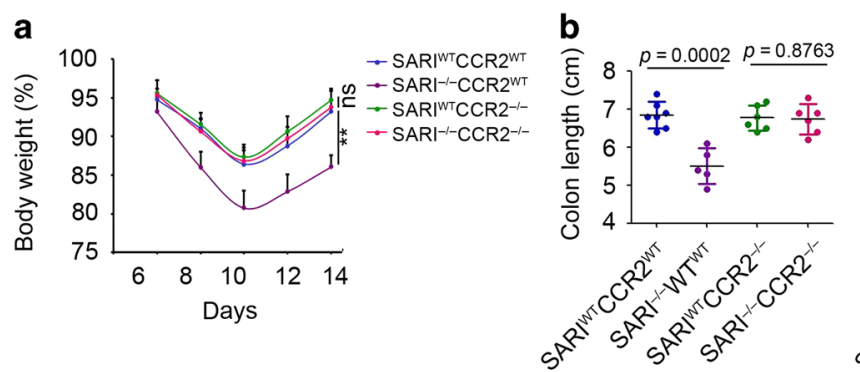

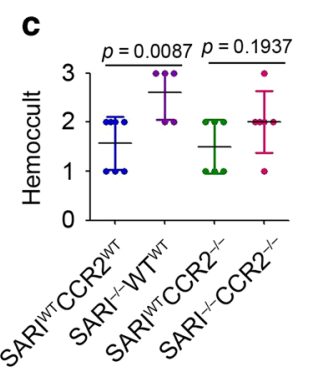

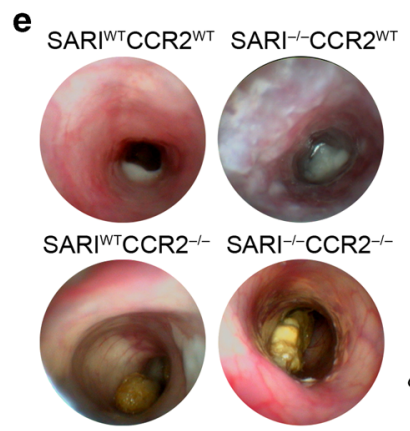
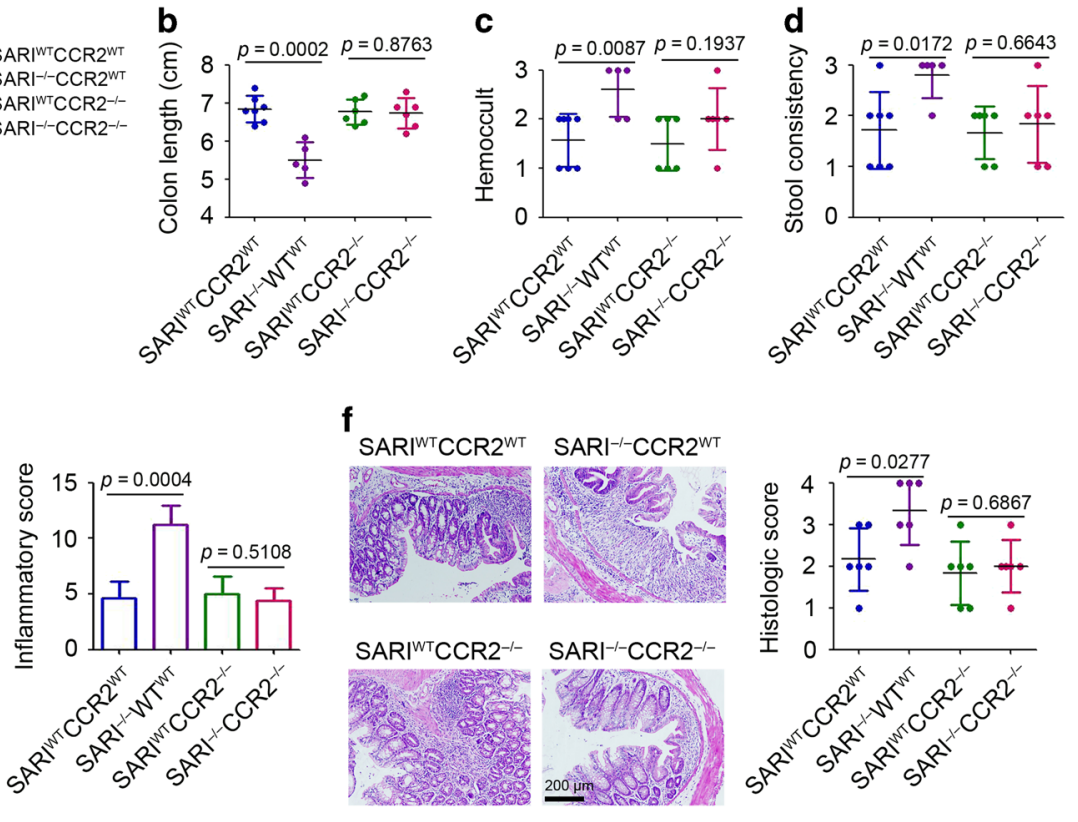

Fig. 5 Knockout of CCR2 blocks the promotion role of SARI deficiency on colitis. a Weight change analysis of $S A R I^{\mathrm{WT}} C C R 2^{\mathrm{WT}}, S A R 1^{-1-} C C R 2^{\mathrm{WT}}$, $S A R I^{\mathrm{WT}} C C R 2^{-1-}, S A R I^{-1-} C C R 2^{-1-}$ mice receiving $3 \%$ DSS treatment $\left(n \geq 5,{ }^{* *} p<0.01\right.$; ns, no significant difference). Colon length (b), hemoccult (c) and stool consistency (d) of SARI ${ }^{\mathrm{WT}} C C R 2^{\mathrm{WT}}, \mathrm{SARI}^{-1-} \mathrm{CCR2} 2^{\mathrm{WT}}, \mathrm{SAR}{ }^{\mathrm{WT}} C C R 2^{-1-}, \mathrm{SARI}^{-1-}{ }^{-} C C R 2^{-1-}$ mice receiving $3 \%$ DSS treatment $(n \geq 5)$. Highresolution mini-endoscopy (e) and H\&E images (f) of colon cross-sections and the quantitation of clinical (e) and histological inflammation (f) $(n \geq 5)$

Supplementary Fig. 6b). Similar expression of ceruloplasmin and HIF-1a was also observed in colonic tissues from SARI ${ }^{W T}$ and $\mathrm{SARI}^{-1-}$ mice with 3\% DSS treatment (Supplementary Fig. 6c). In normal conditions, the activation of epithelial STAT1, STAT3, and NF-KB signaling are maintained at a basal level and no significant changes in their levels were found in SARI-deficient mice (Supplementary Fig. 6d). Notably, SARI deficiency promoted STAT1 activation in IECs under colitis (Fig. 6d). In lipopolysaccharide-treated condition, knockdown of SARI promoted p-STAT1 levels in human IECs (Fig. 6e). After treatment with MG132, a specific proteasome inhibitor, SARI-induced reduction of p-STAT1 was blocked (Fig. 6f). To measure the kinetics of p-STAT1 and STAT1 protein degradation, we added cycloheximide to arrest protein synthesis. We observed that both of the p-STAT1 and STAT1 protein were degraded slower in the shSARI-transfected cells than in the control cells (Fig. $6 \mathrm{~g}$, h). Moreover, depleting macrophages had no significant effect on intestinal p-STAT1 and STAT1 expression, whereas $\mathrm{p}-\mathrm{STAT3}$ and NF-KB p65 expression was inhibited (Fig. 6i). Whereas the hyper-activation of STAT1 only in the irradiated $S A R I^{-1-}$ mice receiving $S A R I^{\mathrm{WT}}$ and $S A R I^{-1-} \mathrm{BM}$ (Fig. 6j). These results suggested that SARI directly targets STAT1 and promotes nuclear STAT1 degradation with a proteasedependent manner in IECs.

Inhibition of STAT1 blocks the promoting role of SARI deficiency on acute colitis

To further evaluate the role of STAT1 during colitis regulated by SARI, the STAT1 specific inhibitor, Fludarabine, ${ }^{30}$ was employed to treat $S A R I^{W T}$ and $S A R I^{-1-}$ mice under acute colitis (Fig. 7a; Supplementary Fig. 7a). The analysis of body weight loss, colon length, bleeding, and stool consistency indicated that less severe colitis was present in $S A R I^{\mathrm{WT}}$ and $S A R I^{-1-}$ mice that were treated with Fludarabine (Fig. 7b, c; Supplementary Fig. 7b, c). Representative mini-endoscopic images and histopathological analysis of colon cross-sections showed that less inflammatory injury and colon damage were detected both in Fludarabine-injected SARI ${ }^{\mathrm{WT}}$ and $S A R I^{-1-}$ mice compared with that in $S A R I^{-1-}$ mice injected with DMSO (Fig. 7d, e). Further, there was no significant difference in inflammatory and histopathological scores between $S A R I^{\mathrm{WT}}$ and $S A R I^{-1-}$ mice treated with Fludarabine (Fig. 7d, e). The results of immunofluorescence staining suggested that fewer infiltrating activated macrophages were detected in $S A R I^{\mathrm{WT}}$ and $S A R I^{-1-}$ mice that were treated with Fludarabine (Supplementary Fig. 7d). Furthermore, ELISA analysis suggested that SARI ${ }^{\mathrm{WT}}$ and $S A R I^{-1-}$ mice that were treated with Fludarabine had lower expression of CCL2 and proinflammatory cytokines (Supplementary Fig. 7e, f). These results indicated that STAT1 plays a necessary role during SARI impairing colitis development.

\section{DISCUSSION}

During colitis development, the equilibrium of the intestinal immune microenvironment is altered ${ }^{2}$ and macrophages are one of the necessary initiators and effectors in this process. ${ }^{31}$ Our results show that SARI deficiency facilitated colitis in mice by promoting colon inflammation dependent on macrophages recruitment in colon tissue. Mechanistically, SARI directly targeted STAT1, and hence promoted nuclear STAT1 degradation and impaired colon inflammation-dependent STAT1/CCL2 transcriptional activation in IECs.

Previous evidence demonstrated that IFN-gamma-inducible SARI controls Th17-mediated immunopathology by suppressing IL-23 production during Trypanosoma cruzi infection. ${ }^{23}$ Meanwhile, BATF, another member of BATF family, facilitated the development of IL-23R expressing CD4 ${ }^{+}$Th17 cells, and hence promoted colitis-associated cancer formation. ${ }^{32}$ Adding to these findings, the present study for the first time showed that SARI deficiency enhances acute and chronic colitis in mice. Furthermore, our results indicated that colonic SARI expression was downregulated after 3\% DSS and 2\% DSS treatment at the protein level (data not shown), which may be the cause of colitis in normal mice.

Intestinal epithelial cells are the major cell type that composes colon tissues and are an important regulator of barrier function and immune homeostasis. ${ }^{33,34}$ Our previous study indicated that 
a

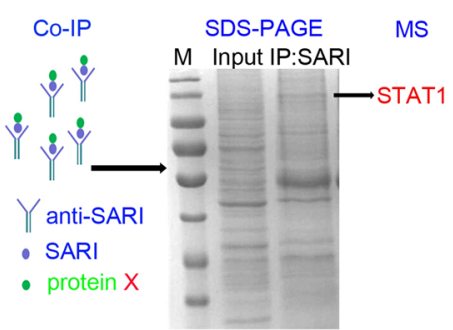

b

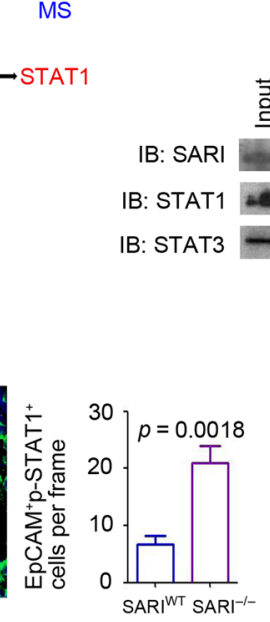

C

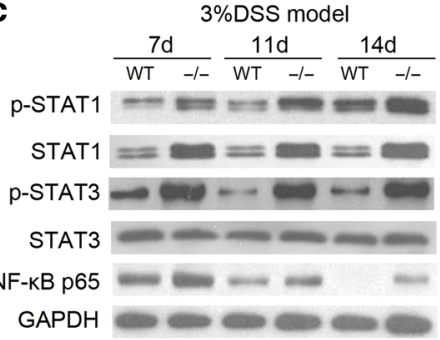

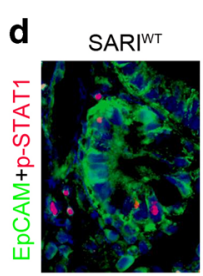
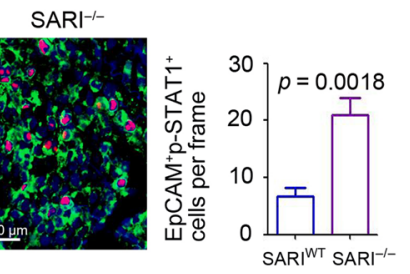

e

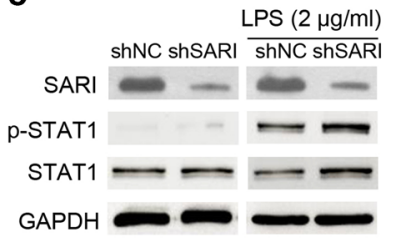

f

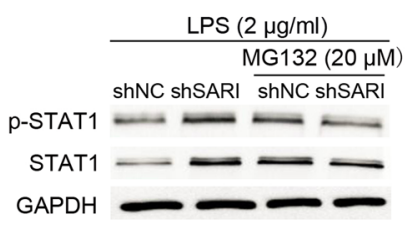

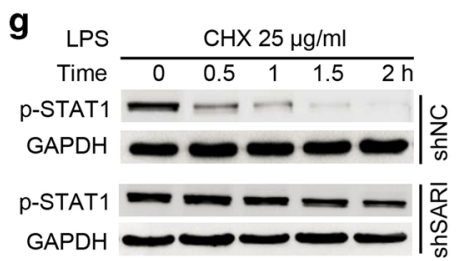

h

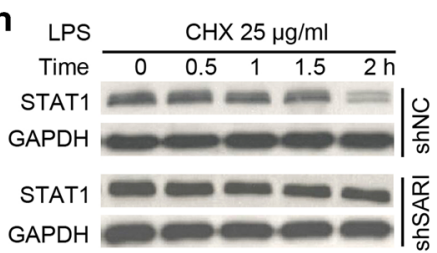

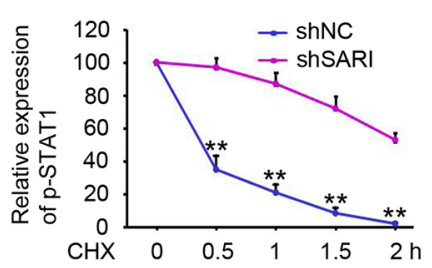

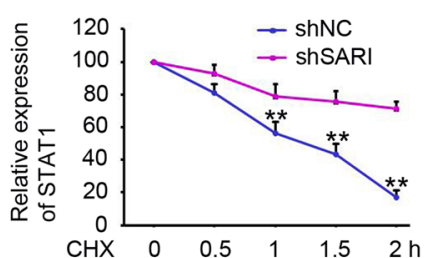

i

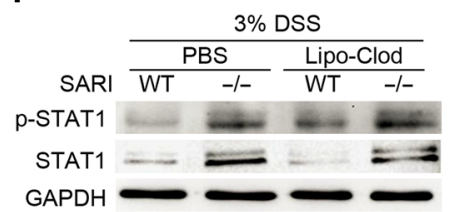

j

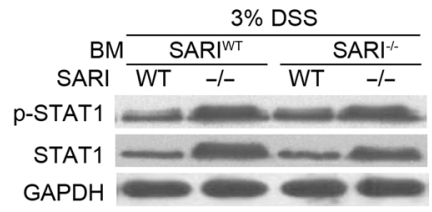

Fig. 6 SARI inhibits CCL2 expression through targeting STAT1 in IECs. a Colonic tissues from 3\% DSS-treated SARI ${ }^{\mathrm{WT}}$ mice (14 d post treatment) were collected for coimmunoprecipitation. The products after adding anti-SARI antibody were separated by SDS-PAGE, and the differential band was analyzed by mass spectrometry (MS). b Coimmunoprecipitation analysis of whole lysates from colitis SARI ${ }^{\mathrm{WT}}$ mice using anti-SARI, anti-STAT1, and anti-STAT3 antibody. c Immunoblots of p-STAT1, STAT1, p-STAT3, STAT3, NF-кB p65 protein expression in whole colonic tissues from $S A R I^{W T}$ and $S A R I^{-1-}$ mice receiving $3 \%$ DSS treatment for 7,11 and 14 days. GAPDH was used as loading control. d Staining for EpCAM (green) and p-STAT1 (red) in colonic tissues from SARI ${ }^{\mathrm{WT}}$ and $S A R I^{-1-}$ mice receiving 3\% DSS treatment for 14 days; DAPI staining for nuclei. The number of EpCAM- and p-STAT1-positive cells were counted and analyzed. e Immunoblots of SARI, p-STAT1, STAT1 protein expression in shNC- or shSARI-transfected IECs with or without LPS $(2 \mu \mathrm{g} / \mathrm{ml})$ treatment for $2 \mathrm{~h}$. GAPDH was used as loading control. $\mathbf{f}$ Immunoblots of SARI, p-STAT1, STAT1 protein expression in shNC- or shSARI-transfected IECs with LPS $(2 \mu \mathrm{g} / \mathrm{ml})$ combining MG132 $(20 \mu \mathrm{M})$ treatment for $2 \mathrm{~h}$. GAPDH was used as loading control. Measurement of p-STAT1 $(\mathbf{g})$ and STAT1 (h) in LPS $(2 \mu \mathrm{g} / \mathrm{ml})$-treated shNC- or shSARI-transfected IECs lysates harvested at $0,0.5,1,1.5$, and $2 \mathrm{~h}$ after the addition of cycloheximide (CHX) to arrest protein synthesis. Densitometric analysis of p-STAT1 expression in multiple western blots after CHX addition $\left(n=3 ;^{* *} p<0.01\right)$. i Immunoblots of p-STAT1, STAT1, p-STAT3 and NF- ${ }^{*} B$ p65 expression in whole colons from SARI ${ }^{\mathrm{WT}}$-PBS, SARI ${ }^{-1-}$-PBS, SARI ${ }^{\mathrm{WT}}$-Lipo clod and SARI ${ }^{-1-}$-Lipo clod mice receiving $3 \%$ DSS treatment for 14 days. $j$ Immunoblots of $\mathrm{p}-\mathrm{STAT} 1$ and STAT1 expression in whole colonic tissues from SARI ${ }^{\mathrm{WT}}$ mice $^{\text {with SARI }}{ }^{\mathrm{WT}} \mathrm{BM}^{\mathrm{B}}\left(\mathrm{BM}{ }^{\mathrm{WT}} \mathrm{mice}^{\mathrm{WT}}\right)$, SARI ${ }^{-I-}$

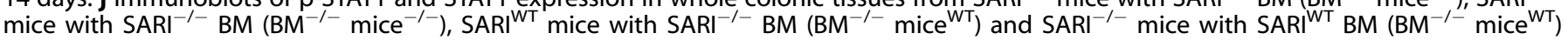
receiving $3 \%$ DSS treatment. GAPDH was used as a loading control

SARI expressed in most epithelial cells from normal colon tissues of patients. ${ }^{20}$ However, whether SARI localized in all colonic epithelial cells of mice was still unclear, which limits to the efficient antibody against m-SARI for IHC staining. Macrophages, myeloid-derived suppressor cells, monocytes, T-cells, dendritic cells, mesenchymal cells, and fibroblasts comprise the immune microenvironment, which responds to colitis, bacterial infection, and tumorigenesis. ${ }^{35,36}$ Our results suggested that IECs, rather than myeloid cells, are the initiators and orchestrators of the enhanced colitis observed in $S A R I^{-1-}$ mice, as evidenced by BM transplantation experiments. However, during colitis, increased infiltration of macrophages, myeloid-derived suppressor cells and monocytes into colon tissues of SARI ${ }^{-1-}$ mice, which exhibited enhanced colitis, accompanied with the upregulation of proinflammatory cytokine expression, was noted. This may contribute to the downstream effects of the change in SARI deletion-induced cytokine secretion in IECs. However, the contribution of SARI function in other cell types to intestinal tumorigenesis remains to be determined.

Recent studies have uncovered that BATF family members have unique, nonredundant, and positive transcriptional activities in dendritic, B- and T cells, and regulate the development of these cells. $^{23} \mathrm{SARI}^{-1-}$ mice have fewer lung-resident $\mathrm{CD} 103^{+} \mathrm{CD} 11 \mathrm{~b}^{-}$ CDCs and $\mathrm{CD}_{103^{-}} \mathrm{CD} 11 \mathrm{~b}^{-}$macrophages than wild-type mice after T. gondii infection, which shows that SARI is involved in the maintenance of these cell subsets during infection. ${ }^{37}$ Our results suggested that the development and infiltration of immune cells, including macrophages, myeloid-derived suppressor cells and monocytes in the colon microenvironment are not affected by $S A R I$ deletion under normal conditions. This provides further 
a

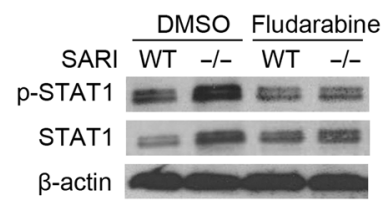

b

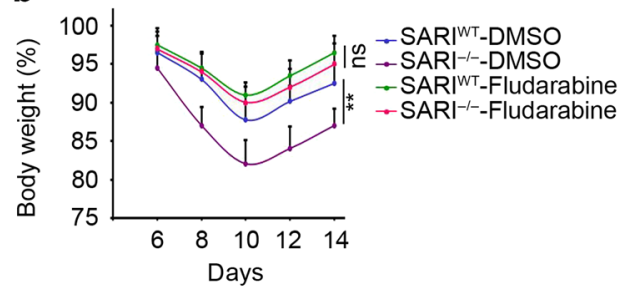

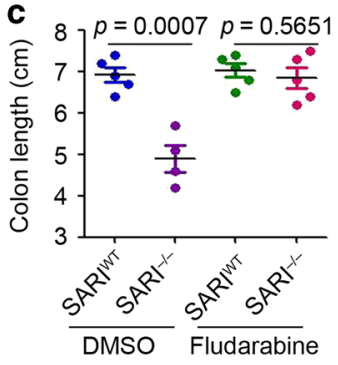

d

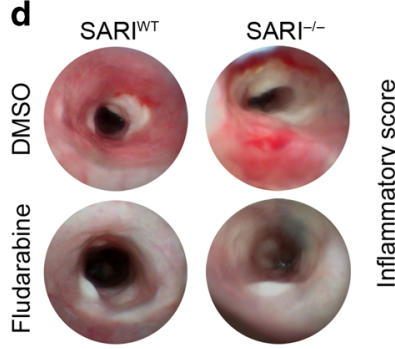

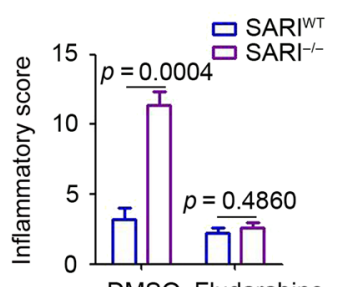

DMSO Fludarabine

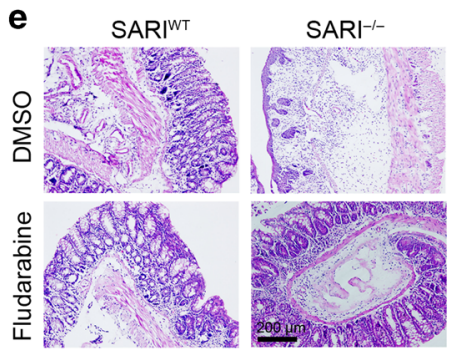

Fig. 7 Inhibition of STAT1 blocks the promotion role of SARI deficiency on colitis. a Immunoblots of p-STAT1 and STAT1 expression in whole colonic tissues from SARI ${ }^{\mathrm{WT}}$-DMSO, SARI ${ }^{\mathrm{WT}}$-Fludarabine, SARI ${ }^{-1}-\mathrm{DMSO}$ and SARI ${ }^{-1}$-Fludarabine mice receiving $3 \%$ DSS treatment. $\beta$-actin was used as a loading control. b Weight change analysis of SARI ${ }^{\mathrm{WT}}$-DMSO, SARI ${ }^{\mathrm{WT}}$-Fludarabine, SARI ${ }^{-1-}$-DMSO and SARI ${ }^{-1-}$-Fludarabine mice receiving $3 \%$ DSS treatment $\left(n \geq 4,{ }^{* *} p<0.01 ;\right.$ ns, no significant difference). c Colon length of SARI ${ }^{\mathrm{WT}}$-DMSO, SARI ${ }^{\mathrm{WT}}$-Fludarabine, SARI ${ }^{\prime \prime-}$ DMSO and SARI ${ }^{-1-}$-Fludarabine mice receiving $3 \%$ DSS treatment $(n \geq 4)$. High-resolution mini-endoscopy (d) and H\&E images (e) of mice for quantitation of clinical (d) and histological inflammation (e) of SARI ${ }^{\mathrm{WT}}$-DMSO, SARI ${ }^{\mathrm{WT}}$-Fludarabine, SARI ${ }^{-1-}$-DMSO and SARI ${ }^{-1-}{ }^{-}$-Fludarabine mice receiving $3 \%$ DSS treatment $(n \geq 4)$

evidence for the need of understanding the role of SARI-mediated paracrine changes in SARI-deficient IECs under colitis. Furthermore, deletion of macrophages also prevents SARI deficiencymediated inflammation upregulation in mice. In conclusion, anticolitis role of SARI was dependent on macrophages.

Chemokines are a family of low molecular weight proteins with an essential role in leukocyte trafficking during both homeostasis and inflammation. ${ }^{38}$ Among them, CCL2 is one of the key chemokines that regulate migration and infiltration of monocytes/macrophages. ${ }^{39}$ During colitis development, the expression of CCL2 was enhanced while a massive infiltration of intracolonic macrophages/monocytes occurred, which is a mediator of colitis. ${ }^{40}$ These observations suggested that targeting CCL2 may be an efficient therapeutic strategy for colitis and its associated cancer. Our study indicated the dramatic upregulation of epithelial $\mathrm{CCL} 2$ expression in $\mathrm{SARI}^{-1-}$ mice under colitis. Inhibition of CCL2CCR2 signaling by inhibitors or knockout of CCR2 could efficiently attenuate the SARI deficiency-mediated severe colitis development, accompanied with less macrophage infiltration. Therefore, we propose that CCL2 plays a necessary role during SARIregulated colitis. This additionally supports the conclusion that CCL2-mediated $\mathrm{CCR}^{+}$macrophage infiltration is the necessary mediator during SARI-regulated colitis.

Key endogenous (intrinsic) factors, including transcription factors (such as NF-KB and STAT3) and major inflammatory cytokines (including IL-1 $\beta$, IL- 6 , and TNF- $\alpha$ ), have been demonstrated to be involved in inflammation and inflammation-related tumorigenesis. ${ }^{29,41,42}$ The present results suggested enhanced expression and activation of these transcription factors and inflammatory cytokines in $S A R I^{-1-}$ mice in the colitis model, which is related to the enhanced colitis in mice. Higher activation of STAT1, STAT3, and NF-KB, which were demonstrated as transcription factor CCL2, were found in the SARI-deficient mice, ${ }^{43-46}$ but the results indicated that only STAT1 is the direct target of SARI. Meanwhile, similar HIF-1a expression was observed in colonic tissues from SARI ${ }^{W T}$ and $S A R I^{-1-}$ mice with 3\% DSS treatment, which may contribute to the no/low level of ceruloplasmin. Thus, we speculated that higher activation of
STAT3 and NF-KB may contribute to the more severe colitis in SARI-deficient mice under colitis condition. Our study also found that SARI inhibited epithelial STAT1 expression through directly targeting nuclear STAT1 and promoting nuclear STAT1 degradation in a protease-dependent manner. Furthermore, in vitro and in vivo results indicated that STAT1 plays a crucial role during CCL2 expression, macrophages infiltration, and colitis regulated by SARI. Therefore, we speculated that the upregulation of STAT3 and $\mathrm{NF}-\mathrm{KB}$ in $S A R I^{-1-}$ mice in colitis is the downstream effect of STAT1 inhibition by SARI. However, our study only demonstrated the protease-dependent degradation of STAT1 by SARI in IECs, but the mechanism behind SARI-driven degradation of STAT1 is still unclear. Thus, further studies are needed to determine the potential synergistic protease during the process of SARI promoting STAT1 degradation. In conclusion, anticolitis role of SARI was STAT1 dependent. The discrepancy in findings between the present study and a previous study by Kanemaru et al. may be attributed to the different experimental model and cells used. ${ }^{21}$

In summary, our study provides evidence for an antiinflammatory role for SARI in mice by promoting STAT1 degradation and negatively regulating STAT1/CCL2 transcriptional activity. The demonstrated regulatory mechanism of intestinal homeostasis by SARI paves the way to understand the role of BATF family proteins in gastrointestinal disorders. Considering the initiation role of colitis in intestinal tumorigenesis, the present and previous study ${ }^{20}$ on the function of SARI in colitis and colon tumorigenesis bears great importance. This may provide novel therapeutic targets for colitis and its associated cancer, and perhaps for other inflammation-associated cancers.

\section{ACKNOWLEDGEMENTS}

Writing assistance was obtained from the Editage Company. This study was supported by the National Natural Science Foundation of China Programme grant (No. 81772939) and the National Key R\&D Programme of China grant (No. 2017YFA0105702) and the National Key R\&D Programme of China (2016YFC1201700) and the Fundamental Research Funds for the Central Universities 
(2017SCU12033) and the Special Foundation of China Postdoctoral Science (2018T110980).

\section{AUTHOR CONTRIBUTIONS}

H.D. and L.D. designed the study; L.D. and H.D. analyzed and interpreted data, drafted and critically revised the manuscript. L.D., Y.L., L.C., H.W. performed most of the experiments with assistance from Y.L., G.S., Z.D., J.L.; P.F., X.H. and Z.Z. collected human colon cancer tissue; Q.W. and X.S. were involved in animal study. S.Z. and Y.Y. provided assistance in technical support, data analysis and interpretation. W.H. and C.P. critically revised the manuscript. L.D., D.Y., Y.W. and H.D. obtained funding and supervised students; all authors read and approved the manuscript.

\section{ADDITIONAL INFORMATION}

The online version of this article (https://doi.org/10.1038/s41385-019-0178-9) contains supplementary material, which is available to authorized users.

Competing interests: The authors declare no competing interests.

Publisher's note: Springer Nature remains neutral with regard to jurisdictional claims in published maps and institutional affiliations.

\section{REFERENCES}

1. Kaplan, G. G. The global burden of IBD: from 2015 to 2025. Nat. Rev. Gastroenterol. Hepatol. 12, 720-727 (2015).

2. Grivennikov, S. I., Greten, F. R. \& Karin, M. Immunity, inflammation, and cancer. Cell 140, 883-899 (2010).

3. Chen, L. et al. NLRP12 attenuates colon inflammation by maintaining colonic microbial diversity and promoting protective commensal bacterial growth. Nat. Immunol. 18, 541-551 (2017).

4. Gupta, J. et al. Dual function of p38alpha MAPK in colon cancer: suppression of colitis-associated tumor initiation but requirement for cancer cell survival. Cancer Cell 25, 484-500 (2014).

5. Gillen, C. D., Walmsley, R. S., Prior, P., Andrews, H. A. \& Allan, R. N. Ulcerative colitis and Crohn's disease: a comparison of the colorectal cancer risk in extensive colitis. Gut 35, 1590-1592 (1994).

6. Coussens, L. M., Zitvogel, L. \& Palucka, A. K. Neutralizing tumor-promoting chronic inflammation: a magic bullet? Science 339, 286-291 (2013).

7. Antonioli, L., Blandizzi, C., Pacher, P. \& Hasko, G. Immunity, inflammation and cancer: a leading role for adenosine. Nat. Rev. Cancer 13, 842-857 (2013).

8. $\mathrm{Wu}, \mathrm{Y}$. et al. The role of autophagy in colitis-associated colorectal cancer. Signal Transduct. Target. Ther. 3, 31 (2018).

9. Liu, T., Zhang, L., Joo, D. \& Sun, S. C. NF-kappaB signaling in inflammation. Signal Transduct. Target. Ther. 2, 17023 (2017).

10. Chen, T. T., Tsai, M. H. \& Kung, J. T. STAT1 regulates marginal zone B cell differentiation in response to inflammation and infection with blood-borne bacteria. J. Exp. Med. 213, 3025-3039 (2016).

11. Ernst, M. et al. STAT3 and STAT1 mediate IL-11-dependent and inflammationassociated gastric tumorigenesis in gp130 receptor mutant mice. J. Clin. Investig. 118, 1727-1738 (2008).

12. Schreiber, S. et al. Activation of signal transducer and activator of transcription (STAT) 1 in human chronic inflammatory bowel disease. Gut 51, 379-385 (2002).

13. Chiriac, M. T. et al. Activation of epithelial signal transducer and activator of transcription 1 by interleukin 28 controls mucosal healing in mice with colitis and is increased in mucosa of patients with inflammatory bowel disease. Gastroenterology 153, 123-138.e128 (2017).

14. Takahashi, R. et al. SOCS1 is essential for regulatory T cell functions by preventing loss of Foxp3 expression as well as IFN-\{gamma\} and IL-17A production. J. Exp. Med. 208, 2055-2067 (2011).

15. Choi, S. H. et al. Synthetic triterpenoid induces 15-PGDH expression and suppresses inflammation-driven colon carcinogenesis. J. Clin. Investig. 124, 2472-2482 (2014).

16. Su, Z. Z. et al. Cloning and characterization of SARI (suppressor of AP-1, regulated by IFN). Proc. Natl Acad. Sci. USA 105, 20906-20911 (2008).

17. Murphy, T. L., Tussiwand, R. \& Murphy, K. M. Specificity through cooperation: BATF-IRF interactions control immune-regulatory networks. Nat. Rev. Immunol. 13, 499-509 (2013).

18. Dash, R. et al. Inhibition of AP-1 by SARI negatively regulates transformation progression mediated by CCN1. Oncogene 29, 4412-4423 (2010).
19. Dash, R. et al. Novel mechanism of MDA-7/IL-24 cancer-specific apoptosis through SARI induction. Cancer Res. 74, 563-574 (2014).

20. Dai, L. et al. SARI inhibits angiogenesis and tumour growth of human colon cancer through directly targeting ceruloplasmin. Nat. Commun. 7, 11996 (2016).

21. Kanemaru, $\mathrm{H}$. et al. Antitumor effect of Batf2 through IL-12p40 up-regulation in tumor-associated macrophages. Proc. Natl Acad. Sci. USA 114, E7331-E7340 (2017).

22. Roy, S. et al. Batf2/Irf1 induces inflammatory responses in classically activated macrophages, lipopolysaccharides, and mycobacterial infection. J. Immunol. 194, 6035-6044 (2015).

23. Kitada, S. \& Kayama, H. BATF2 inhibits immunopathological Th17 responses by suppressing II23a expression during Trypanosoma cruzi infection. J. Exp. Med. 214, 1313-1331 (2017).

24. Wirtz, S. et al. Chemically induced mouse models of acute and chronic intestinal inflammation. Nat. Protoc. 12, 1295-1309 (2017).

25. Xiao, Z. et al. The pivotal role of IKKalpha in the development of spontaneous lung squamous cell carcinomas. Cancer Cell 23, 527-540 (2013).

26. Jenkins, S. J. et al. Local macrophage proliferation, rather than recruitment from the blood, is a signature of TH2 inflammation. Science 332, 1284-1288 (2011).

27. Chen, W. et al. Bindarit, an inhibitor of monocyte chemotactic protein synthesis, protects against bone loss induced by chikungunya virus infection. J. Virol. 89, 12232 (2015).

28. Grivennikov, S. et al. IL- 6 and Stat3 are required for survival of intestinal epithelial cells and development of colitis-associated cancer. Cancer Cell 15, 103-113 (2009).

29. Cooks, T. et al. Mutant $\mathrm{p} 53$ prolongs NF-kappaB activation and promotes chronic inflammation and inflammation-associated colorectal cancer. Cancer Cell 23, 634-646 (2013).

30. Feng, Z. et al. Fludarabine inhibits STAT1-mediated up-regulation of caspase-3 expression in dexamethasone-induced osteoblasts apoptosis and slows the progression of steroid-induced avascular necrosis of the femoral head in rats. Apoptosis: Int. J. Program. Cell Death 22, 1001-1012 (2017).

31. Mantovani, A., Marchesi, F., Malesci, A., Laghi, L. \& Allavena, P. Tumour-associated macrophages as treatment targets in oncology. Nat. Rev. Clin. Oncol. 14, 399-416 (2017).

32. Punkenburg, E. et al. Batf-dependent Th17 cells critically regulate IL-23 driven colitis-associated colon cancer. Gut 65, 1139-1150 (2016).

33. Goto, Y., Uematsu, S. \& Kiyono, H. Epithelial glycosylation in gut homeostasis and inflammation. Nat. Immunol. 17, 1244-1251 (2016).

34. Peterson, L. W. \& Artis, D. Intestinal epithelial cells: regulators of barrier function and immune homeostasis. Nat. Rev. Immunol. 14, 141-153 (2014).

35. Nowarski, R., Jackson, R. \& Flavell, R. A. The stromal intervention: regulation of immunity and inflammation at the epithelial-mesenchymal barrier. Cell 168, 362-375 (2017).

36. Veldhoen, M. \& Brucklacher-Waldert, V. Dietary influences on intestinal immunity. Nat. Rev. Immunol. 12, 696-708 (2012).

37. Tussiwand, R. et al. Compensatory dendritic cell development mediated by BATFIRF interactions. Nature 490, 502-507 (2012).

38. Nagarsheth, N., Wicha, M. S. \& Zou, W. Chemokines in the cancer microenvironment and their relevance in cancer immunotherapy. Nat. Rev. Immunol. 17, 559-572 (2017).

39. Qian, B. Z. et al. CCL2 recruits inflammatory monocytes to facilitate breast-tumour metastasis. Nature 475, 222-225 (2011).

40. Kim, Y. G. et al. The Nod2 sensor promotes intestinal pathogen eradication via the chemokine CCL2-dependent recruitment of inflammatory monocytes. Immunity 34, 769-780 (2011).

41. Diakos, C. I., Charles, K. A., McMillan, D. C. \& Clarke, S. J. Cancer-related inflammation and treatment effectiveness. Lancet Oncol. 15, e493-e503 (2014).

42. Di Paolo, N. C. \& Shayakhmetov, D. M. Interleukin 1alpha and the inflammatory process. Nat. Immunol. 17, 906-913 (2016).

43. Hu, X., Park-Min, K. H., Ho, H. H. \& Ivashkiv, L. B. IFN-gamma-primed macrophages exhibit increased CCR2-dependent migration and altered IFN-gamma responses mediated by Stat1. J. Immunol. 175, 3637-3647 (2005).

44. Lee, H. Y. et al. Sphingosylphosphorylcholine stimulates CCL2 production from human umbilical vein endothelial cells. J. Immunol. 186, 4347-4353 (2011).

45. Izumi, K. et al. Targeting the androgen receptor with siRNA promotes prostate cancer metastasis through enhanced macrophage recruitment via CCL2/CCR2induced STAT3 activation. EMBO Mol. Med. 5, 1383-1401 (2013).

46. Bauer, D. et al. Diallyl disulfide inhibits TNFalpha induced CCL2 release through MAPK/ERK and NF-Kappa-B signaling. Cytokine 75, 117-126 (2015) 